\title{
ON FINITE GK-DIMENSIONAL NICHOLS ALGEBRAS OF DIAGONAL TYPE
}

\author{
NICOLÁS ANDRUSKIEWITSCH, IVÁN ANGIONO, ISTVÁN HECKENBERGER
}

\begin{abstract}
It was conjectured in arXiv:1606.02521 that a Nichols algebra of diagonal type with finite Gelfand-Kirillov dimension has finite (generalized) root system. We prove the conjecture assuming that the rank is 2. We also show that a Nichols algebra of affine Cartan type has infinite Gelfand-Kirillov dimension.
\end{abstract}

\section{INTRODUCTION}

In this paper we contribute to the classification of Hopf algebras with finite Gelfand-Kirillov dimension, GKdim for short. Specifically we propose:

Conjecture 1.1. [AAH, Conjecture 1.5] If $V$ is a braided vector space of diagonal type and dimension $\theta \in \mathbb{N}$ such that the GKdim of its Nichols algebra $\mathcal{B}(V)$ is finite, then its (generalized) root system is finite.

Since the classification of the Nichols algebras of diagonal type with finite generalized root system is known [H2], a positive answer to the Conjecture 1.1 would imply the classification of the Nichols algebras of diagonal type with finite GKdim. Indeed, the converse in the Conjecture is clearly true. The defining relations of these Nichols algebras are also known An1. The GKdim of these Nichols algebras can be computed (2.6). Our main result provides partial answers to this Conjecture:

Theorem 1.2. Conjecture 1.1 holds in the following cases:

(a) $V$ is of affine Cartan type.

(b) $\theta=2$.

We collect some necessary definitions and concepts in Section 2, Section 3 is devoted to general results that might be of interest elsewhere. Part (a) is proved in Proposition 3.1. Part (b), which is Theorem 4.1, is proved in 4.2. after some preparatory Lemmas in 4.1 .

2000 Mathematics Subject Classification. 16W30.

The work of N. A. and I. A. was partially supported by CONICET, Secyt (UNC), the MathAmSud project GR2HOPF. The work of I. A. was partially supported by ANPCyT (Foncyt). The work of N. A., respectively I. A., was partially done during a visit to the University of Marburg, respectively the MPI (Bonn), both visits supported by the Alexander von Humboldt Foundation. 


\section{Preliminaries}

2.1. Conventions. Let $\mathbb{k}$ be an algebraically closed field of characteristic zero. All the vector spaces, algebras and tensor products are over $\mathbb{k}$. Let $\mathbb{N}=\{1,2, \ldots\}, \mathbb{N}_{0}=\mathbb{N} \cup 0$. Given $\theta \in \mathbb{N}$, we set $\mathbb{I}_{\theta}=\{1,2, \ldots, \theta\}$, or simply $\mathbb{I}$ if $\theta$ is clear from the context. In the polynomial ring $\mathbb{Z}[\mathrm{q}]$, we denote

$$
(n)_{\mathrm{q}}=\sum_{j=0}^{n-1} \mathrm{q}^{j}, \quad(n)_{\mathrm{q}}^{!}=\prod_{j=1}^{n}(j)_{\mathrm{q}}, \quad n \in \mathbb{N}_{0} .
$$

If $q \in \mathbb{k}$, then $(n)_{q},(n)_{q}^{!}$are the evaluations at $q$.

We denote by $\widehat{\Gamma}$ the group of multiplicative characters (one-dimensional representations) of a group $\Gamma$. Let $\mathbb{G}_{N}$ be the group of roots of unity of order $N, \mathbb{G}_{N}^{\prime}$ the subset of primitive roots of order $N$, and $\mathbb{G}_{\infty}=\bigcup_{N \in \mathbb{N}} \mathbb{G}_{N}$.

Let $H$ be a Hopf algebra (always with bijective antipode). A braided Hopf algebra means a Hopf algebra in the category ${ }_{H}^{H} \mathcal{Y} \mathcal{D}$ of Yetter-Drinfeld modules over $H$. If $R$ is a Hopf algebra in ${ }_{H}^{H} \mathcal{Y} \mathcal{D}$, then $R \# H$ is the bosonization of $R$ by $H$. Let ad be the adjoint action of $R \# H$ and $\operatorname{ad}_{c}$ the braided adjoint action of $R$. Then $\operatorname{ad}_{c} x \otimes \mathrm{id}=\operatorname{ad}(x \# 1)$ for $x \in R$. If $x \in R$ is primitive, then $\operatorname{ad}_{c} x(y)=x y-$ multiplication $\circ c(x \otimes y)$ for all $y \in R$.

If $(V, c)$ is a braided vector space, then $\mathcal{B}(V)=T(V) / \mathcal{J}(V)$ is the Nichols algebra of $V$, see [AS1, A, AA2 for surveys on this notion.

A braided vector space of diagonal type is a pair $(V, c)$, where $V$ is a vector space of dimension $\theta$ with a basis $\left(x_{i}\right)_{i \in \mathbb{I}_{\theta}}$, and the braiding $c \in G L(V \otimes V)$ is given by $c\left(x_{i} \otimes x_{j}\right)=q_{i j} x_{j} \otimes x_{i}$ for all $i, j \in \mathbb{I}$; here $\mathbf{q}=\left(q_{i j}\right)_{i, j \in \mathbb{I}_{\theta}} \in\left(\mathbb{k}^{\times}\right)^{\theta \times \theta}$, $q_{i i} \neq 1$. Let

$$
\widetilde{q}_{i j}:=q_{i j} q_{j i}, \quad i \neq j \in \mathbb{I}_{\theta} .
$$

The generalized Dynkin diagram of $\mathbf{q}$ is a graph with set of points $\mathbb{I}_{\theta}$ with the vertex $i$ decorated with $q_{i i}$; and for $i \neq j \in \mathbb{I}_{\theta}$, no edge between $i$ and $j$ when $\widetilde{q}_{i j}=1$, otherwise there is an edge decorated with $\widetilde{q}_{i j}$.

2.2. The Gelfand-Kirillov dimension. A comprehensive exposition is $\mathrm{KL}$. For further use, we recall statements from $\mathrm{AAH}, \S 2.3 .2]$ inspired by [R, Lemma 19].

Lemma 2.1. Let $\mathcal{B}=\oplus_{n>0} \mathcal{B}^{n}$ be a finitely generated graded algebra with $\mathcal{B}^{0}=\mathbb{k}$. Let $\left(y_{k}\right)_{k \geq 0}$ be a family of homogeneous elements of $\mathcal{B}$ such that

$$
\left(y_{i_{1}} \ldots y_{i_{l}}: i_{j} \in \mathbb{N}, i_{1}<\cdots<i_{l}\right)
$$

is a family of linearly independent elements. If there exist $m, p \in \mathbb{N}$ such that $\operatorname{deg} y_{i} \leq m i+p$, for all $i \in \mathbb{N}$, then $\operatorname{GKdim} \mathcal{B}=\infty$. 
The following Lemma is due to Rosso [ $\underline{\mathrm{R}}$, Lemma 14, Corollary 18]. Let $(U, c)$ be a braided vector space of diagonal type, with respect to a basis $x_{1}, x_{2}$ and a matrix $\left(q_{i j}\right)_{i, j \in \mathbb{I}_{2}}$. We set

$$
\mu_{k}=\prod_{i=0}^{k-1}\left(1-q_{11}^{i} \widetilde{q}_{12}\right), \quad y_{k}=\left(\operatorname{ad}_{c} x_{1}\right)^{k} x_{2} .
$$

Lemma 2.2. (a) If $k \in \mathbb{N}$, then $y_{k}=0$ iff $\mu_{k} x_{1}^{k}=0$, iff $(k)_{q_{11}} ! \mu_{k}=0$.

(b) If $y_{k} \neq 0$ for all $k \in \mathbb{N}$, then the set (2.1) is linearly independent.

(c) If $(k)_{q_{11}} ! \mu_{k} \neq 0$ for every $k \in \mathbb{N}$, then $\operatorname{GKdim} \mathcal{B}(U)=\infty$.

(d) If $q_{11}=1$ and $\widetilde{q}_{12} \neq 1$, then $\operatorname{GKdim} \mathcal{B}(U)=\infty$.

2.3. Nichols algebras of diagonal type. We fix a braided vector space of diagonal type $(V, c)$ with notation as in $\$ 2.1$. We assume that the generalized Dynkin diagram is connected.

Let $\left(\alpha_{i}\right)_{i \in \mathbb{I}}$ be the canonical basis of $\mathbb{Z}^{\theta}$. Let $\mathbf{q}$ be the bicharacter on $\mathbb{Z}^{\theta}$ such that $\mathbf{q}\left(\alpha_{i}, \alpha_{j}\right)=q_{i j}$ for all $i, j \in \mathbb{I}$; we set $q_{\alpha \beta}=\mathbf{q}(\alpha, \beta)$ for $\alpha, \beta \in \mathbb{Z}^{\theta}$.

Then $T(V)$ and $\mathcal{B}(V)$ are $\mathbb{Z}^{\theta}$-graded with $\operatorname{deg} x_{i}=\alpha_{i}$ for all $i \in \mathbb{I}$. For each $\alpha \in \mathbb{Z}^{\theta}, \mathcal{B}^{\alpha}(V)$ denotes the homogeneous component of degree $\alpha$.

Let $\Gamma=\mathbb{Z}^{\theta}$. We realize $V$ in $\underset{\mathbb{k} \Gamma}{\mathbb{k} \Gamma} \mathcal{Y} \mathcal{D}$ by choosing the family $\left(\chi_{i}\right)_{i \in \mathbb{I}}$ in $\widehat{\Gamma}$ such that $\chi_{j}\left(\alpha_{i}\right)=q_{i j}$ for all $i, j \in \mathbb{I}$. Then $\mathcal{B}(V)$ becomes an $\mathbb{Z}^{\theta}$-graded object in $\underset{\mathbb{k} \Gamma}{\mathbb{k} \Gamma} \mathcal{Y} \mathcal{D}$. There are skew-derivations $\partial_{i}, i \in \mathbb{I}$ of $\mathcal{B}(V)$, such that $\partial_{i}\left(x_{j}\right)=\delta_{i j}$ and

$$
\partial_{i}(x y)=x \partial_{i}(y)+\partial_{i}(x)\left(\alpha_{i} \cdot y\right), \quad x, y \in \mathcal{B}(V) .
$$

Let $\mu_{k}, y_{k}=\left(\operatorname{ad}_{c} x_{1}\right)^{k} x_{2}$ as in (2.2). We notice that

$$
\partial_{1}\left(y_{k}\right)=0, \quad \partial_{2}\left(y_{k}\right)=\mu_{k} x_{1}^{k}, \quad \text { for all } k \in \mathbb{N}_{0} .
$$

Also, it is well-known, and easy to check by a recursive argument, that

$$
\Delta\left(y_{k}\right)=y_{k} \otimes 1+\sum_{i=0}^{k}\left(\begin{array}{c}
k \\
i
\end{array}\right)_{q_{11}} \frac{\mu_{k}}{\mu_{i}} x_{1}^{k-i} \otimes y_{i} .
$$

By $\left[\mathrm{Kh}\right.$, there is a totally ordered subset $L \subset \mathcal{B}(V)$ consisting of $\mathbb{Z}^{\theta}$ homogeneous elements such that

$$
\left\{\ell_{1}^{m_{1}} \cdots \ell_{k}^{m_{k}} \mid k \in \mathbb{N}_{0}, \ell_{1}>\cdots>\ell_{k} \in L, 0<m_{i}<N_{\ell_{i}} \text { for all } i \in \mathbb{I}_{k}\right\}
$$

is a linear basis of $\mathcal{B}(V)$ (a so called restricted PBW basis); here

$$
N_{\ell}=\min \left\{n \in \mathbb{N}:(n)_{q_{\operatorname{deg} \ell, \operatorname{deg} \ell}}=0\right\} \in \mathbb{N} \cup \infty
$$

is called the height of $\ell$.

Lemma 2.3. If $\mathcal{B}(V)$ has a restricted homogeneous $P B W$ basis with infinitely many $P B W$ generators of infinite height, then $\operatorname{GKdim} \mathcal{B}(V)=\infty$. 
Proof. By assumption there exists $L$ as above and $I \subseteq L$ infinite such that $N_{\ell}=\infty$ for all $\ell \in I$. Let $d \in \mathbb{N}, F_{d} \subset I$ with $\left|F_{d}\right|=d$ and $V_{d}$ the subspace generated by 1 , the $x_{i}$ 's and all $\ell \in F_{d}$. Then $\left(V_{d}\right)^{n+1}$ contains the ordered monomials in $F_{d}$ of degree $\leq n+1$, hence $\operatorname{dim}\left(V_{d}\right)^{n+1} \geq\left(\begin{array}{c}n+d \\ d\end{array}\right)$. Hence $\limsup \log _{n} \operatorname{dim}\left(V_{d}\right)^{n+1} \geq d$. Since $d$ is arbitrary, $\operatorname{GKdim} \mathcal{B}(V)=\infty$.

Assume that $L$ is finite (the Conjecture 1.1 says that this is the case when $\operatorname{GKdim} \mathcal{B}(V)<\infty)$. By [KL, Theorem 12.6.2], we conclude that

$$
\operatorname{GKdim} \mathcal{B}(V)=\left|\left\{\ell \in L: N_{\ell}=\infty\right\}\right| \text {. }
$$

Let $\boldsymbol{\Delta}_{+}^{V}=\boldsymbol{\Delta}_{+}=(\operatorname{deg} \ell)_{\ell \in L}$ be the family of positive roots of $\mathcal{B}(V)$ (with multiplicities). By [HS, Lemma 4.7], it is uniquely determined, i.e. it does not depend on $L$.

We say that we can reflect $V$ at $i \in \mathbb{I}$ if, for all $j \neq i$, there exists $n \in \mathbb{N}_{0}$ such that $(n+1)_{q_{i i}}\left(1-q_{i i}^{n} q_{i j} q_{j i}\right)=0$. In such case, following [H1] we define a generalized Cartan matrix $\left(c_{i j}\right)$ by $c_{i i}=2$ and

$$
c_{i j}:=-\min \left\{n \in \mathbb{N}_{0}:(n+1)_{q_{i i}}\left(1-q_{i i}^{n} q_{i j} q_{j i}\right)=0\right\}, \quad j \neq i .
$$

Let $s_{i} \in G L\left(\mathbb{Z}^{\theta}\right)$ be given by

$$
s_{i}\left(\alpha_{j}\right)=\alpha_{j}-c_{i j} \alpha_{i}, \quad j \in \mathbb{I} .
$$

The reflection at the vertex $i$ of $\mathbf{q}$ is the matrix $\mathcal{R}^{i}(\mathbf{q})=\left(t_{j k}\right)_{j, k \in \mathbb{I}}$, where

$$
t_{j k}:=q_{s_{i}\left(\alpha_{j}\right), s_{i}\left(\alpha_{k}\right)}=q_{j k} q_{i k}^{-c_{i j}} q_{j i}^{-c_{i k}} q_{i i}^{c_{i j} c_{i k}}, \quad j, k \in \mathbb{I} .
$$

Let $\mathcal{R}^{i}(V)$ be the braided vector space of diagonal type with matrix $\mathcal{R}^{i}(\mathbf{q})$.

Theorem 2.4. [H1, AA1] $\operatorname{GKdim} \mathcal{B}\left(\mathcal{R}^{i}(V)\right)=\operatorname{GKdim} \mathcal{B}(V)$.

We say that $V$ admits all reflections if we can reflect $V$ at every $i_{1} \in$ $\mathbb{I}$, then we can reflect $\mathcal{R}^{i_{1}}(V)$ at every $i_{2} \in \mathbb{I}$ and so on, we can reflect $\mathcal{R}^{i_{k}} \ldots \mathcal{R}^{i_{1}}(V)$ at every $i_{k+1} \in \mathbb{I}$ for all $k$.

If $V$ admits all reflections, then we denote by $\mathcal{X}$ the collection of all braided vector spaces of diagonal type obtained from $V$ by a finite number of successive reflections at various vertices. Here any two braided vector spaces with the same braiding matrix are identified. The collection $\left(\boldsymbol{\Delta}_{+}^{U}\right)_{U \in \mathcal{X}}$ is the generalized root system of $V$.

Remark 2.5. If $\operatorname{GKdim} \mathcal{B}(V)<\infty$, then we can reflect $V$ at every $i \in \mathbb{I}$ by Lemma 2.2 (c), Hence $V$ admits all reflections by Theorem 2.4 .

\section{General Results}

Recall that $(V, c)$ is of Cartan type if there exist $a_{i j} \in \mathbb{Z}_{\leq 0}$ such that

$$
q_{i j} q_{j i}=q_{i i}^{a_{i j}}, \quad i \neq j \in \mathbb{I} .
$$

Set $a_{i i}=2, i \in \mathbb{I}$. If $q_{i i} \in \mathbb{G}_{\infty}$, then we choose $a_{i j} \in\left(-\operatorname{ord} q_{i i}, 0\right]$, when $i \neq j$; otherwise it is uniquely determined. In any case, $\mathbf{a}=\left(a_{i j}\right)_{i, j \in \mathbb{I}_{\theta}}$ is 
an indecomposable symmetrizable generalized Cartan matrix $[\mathrm{K}]$. These matrices are of three types: finite, affine or indefinite. If $(V, c)$ is of Cartan type and $\operatorname{GK} \operatorname{dim} \mathcal{B}(V)<\infty$, then Conjecture 1.1 predicts that a is of finite type. Here is the confirmation for the affine type.

Proposition 3.1. If a is of affine type, then $\operatorname{GKdim} \mathcal{B}(V)=\infty$.

Proof. Let $\boldsymbol{\Delta}^{\text {re }}$ denote the set of real roots corresponding to a. There exists a positive imaginary root $\delta$ such that $\boldsymbol{\Delta}^{\mathrm{re}}+\delta=\boldsymbol{\Delta}^{\mathrm{re}} \mathrm{K}$, Proposition $6.3 \mathrm{~d})]$. Let $m$ be the height of $\delta$ and let $\alpha$ be a simple root. Choose a homogeneous restricted $\mathrm{PBW}$ basis of $\mathcal{B}(V)$. Then for all $k \geq 0$ there exists a PBW generator $y_{k}$ of degree $\alpha+k \delta$, hence deg $y_{k}=m k+1$. Therefore $\operatorname{GKdim} \mathcal{B}(V)=\infty$ by Lemma 2.1

An indecomposable generalized Cartan matrix is compactly hyperbolic if it is of indefinite type and every proper minor is of finite type. If $\mathbf{a} \in \mathbb{Z}^{\theta \times \theta}$ is compactly hyperbolic, then $\theta \leq 5$. In fact, the classification of compactly hyperbolic generalized Cartan matrices is known $\mathrm{C}+$; there are the matrices $\left(\begin{array}{ll}2 & a \\ b & 2\end{array}\right)$ with $a b>4 ; 31$ matrices in $\mathbb{Z}^{3 \times 3} ; 3$ matrices in $\mathbb{Z}^{4 \times 4} ; 1$ matrix in $\mathbb{Z}^{5 \times 5}$. To prove Conjecture 1.1 in the Cartan case, it would be enough to verify it for compactly hyperbolic generalized Cartan matrices with $3 \leq \theta \leq$ 5 , as the case $\theta=2$ is taken care by Theorem 4.1 .

Back to the general diagonal type, we distinguish three classes of Nichols algebras. Given $\mathbf{q}$ as above, we say that

$\circ \mathbf{q}$ is of torsion class if $q_{i i}, q_{i j} q_{j i} \in \mathbb{G}_{\infty}$ for all $i \neq j \in \mathbb{I}$;

$\circ \mathbf{q}$ is generic, if $q_{i i} \notin \mathbb{G}_{\infty}$, and $q_{i j} q_{j i}=1$ or $q_{i j} q_{j i} \notin \mathbb{G}_{\infty}$, for all $i \neq j \in \mathbb{I}$.

$\circ \mathbf{q}$ is semigeneric if it is neither generic nor of torsion class.

Remark 3.2.

(1) If $\mathbf{q}$ is of torsion class, then Conjecture 1.1 says that $\operatorname{GKdim} \mathcal{B}(V)<\infty$ implies $\mathrm{GKdim} \mathcal{B}(V)=0$. Indeed all roots are real by $[\mathrm{CH}]$, and they would have finite non-trivial order by Lemma $2.2(\mathrm{~d})$, hence (2.6) applies.

(2) If $\mathbf{q}$ is of torsion class, then the set $\mathcal{X}$ defined after Theorem 2.4 is finite. Indeed, there are finitely many matrices with the shape (2.9).

(3) [R, AA1] If $\mathbf{q}$ is generic, then $\operatorname{GKdim} \mathcal{B}(V)<\infty$ if and only if there exists a Cartan matrix of finite type $\mathbf{a}=\left(a_{i j}\right)$, with symmetrizing diagonal matrix $\left(d_{i}\right)$, and $q \notin \mathbb{G}_{\infty}$ such that $q_{i i}=q^{2 d_{i}}$ and $q_{i j} q_{j i}=q^{2 d_{i} a_{i j}}$ for all $i \neq j \in \mathbb{I}$. Thus Conjecture 1.1 holds in this case.

(4) A semigeneric matrix with finite generalized root system is either of super type or else one of two exceptions of ranks 2 and 4 :

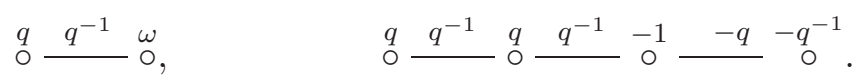


Here $\omega \in \mathbb{G}_{3}^{\prime}$ and $q \notin \mathbb{G}_{\infty}$; the first corresponds to Yamane's exotic quantum groups $[\mathrm{Y}]$ while the second is the row 14 in [H2, Table 3].

3.1. Semigeneric diagonal type. Let us fix $\mathbf{q}=\left(q_{i j}\right)_{i, j \in \mathbb{I}}$ semigeneric with $\operatorname{GKdim} \mathcal{B}(V)<\infty$. Let $\mathbb{J}=\left\{i \in \mathbb{I}: q_{i i} \notin \mathbb{G}_{\infty}\right\}$ be the set of generic points of $\mathbf{q}$ and let $\mathbb{J}_{1}, \ldots \mathbb{J}_{t}$ be the connected components of the generalized Dynkin diagram $\mathbf{q}=\left(q_{i j}\right)_{i, j \in \mathbb{J}}$.

Lemma 3.3. If $i \in \mathbb{I}$ and $j \in \mathbb{J}$, then there exists $h \in \mathbb{N}_{0}$ such that $q_{j j}^{-h}=$ $q_{i j} q_{j i}$. In particular, either $q_{i j} q_{j i}=1$ or $\notin \mathbb{G}_{\infty}$.

Proof. By Lemma 2.2.

Lemma 3.4. If $i \notin \mathbb{J}, j \in \mathbb{J}$ and $q_{i j} q_{j i} \neq 1$, then either ord $q_{i i}=2$ and $q_{i j} q_{j i}=q_{j j}^{-h}$ with $h \in \mathbb{I}_{2}$; or else ord $q_{i i}=3$ and $q_{i j} q_{j i}=q_{j j}^{-1}$.

Proof. First, there exists $h \in \mathbb{N}$ such that $q_{j j}^{-h}=q_{i j} q_{j i}$ by Lemma 3.3. Let $N=\operatorname{ord} q_{i i}$. We apply the reflection at $i$ :

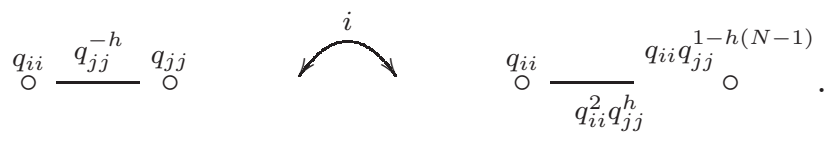

Then either $1=h(N-1)$ that gives $h=1, N=2$; or else there exists $t \in \mathbb{N}$ such that $\left(q_{i i} q_{j j}^{1-h(N-1)}\right)^{-t}=q_{i i}^{2} q_{j j}^{h}$ by Lemma 3.3. A straightforward analysis yields the claim.

As a consequence we derive the corresponding version of Theorem 4.1 for semigeneric braidings. It will be useful for the proof of the general case.

Corollary 3.5. Let $V$ be a braided vector space of semigeneric diagonal type and dimension 2 such that the GKdim of its Nichols algebra $\mathcal{B}(V)$ is finite. Then its generalized root system is finite.

Proof. We may assume that $q_{11} \in \mathbb{G}_{\infty}, q_{22} \notin \mathbb{G}_{\infty}$ up to reflection. Indeed, if neither $q_{11}$ nor $q_{22}$ belong to $\mathbb{G}_{\infty}$, then $V$ is generic by Lemma 2.2 (c), So, either $q_{11} \in \mathbb{G}_{\infty}$ or else $q_{22} \in \mathbb{G}_{\infty}$. If both belong to $\mathbb{G}_{\infty}$, then $q_{i j} q_{j i} \notin \mathbb{G}_{\infty}$. Applying reflection at 1 , we have that the new $q_{22} \notin \mathbb{G}_{\infty}$.

By Lemma 3.4, the Dynkin diagram of $V$ is one of the following:

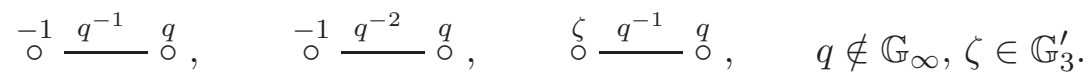

All of them appear in [H2, Table 1], so $V$ has a finite root system.

3.2. Nichols algebras of indefinite Cartan type. Let $A$ be an indecomposable generalized Cartan matrix. Let $W$ be the corresponding Weyl group, see $[\mathrm{K}]$.

Lemma 3.6. Assume that $A$ is of indefinite type. Let $Q$ be the root lattice corresponding to $A$ and let $Q_{+} \subset Q$ be the submonoid generated by the simple roots. Then $W \gamma \cap Q_{+}$is infinite for all $\gamma \in Q_{+}-0$. 
Proof. Let $\pi$ denote the set of simple roots. Let $\gamma \in Q_{+}-0$. Assume that $W \gamma \cap Q_{+}$is finite. Let $\beta=\sum_{\alpha \in \pi} c_{\alpha} \alpha \in W \alpha \cap Q_{+}$, where $c_{\alpha} \geq 0$ for all $\alpha \in \pi$, such that $w \gamma-\beta \notin Q_{+}-0$ for all $w \in W$. Then for all $\alpha \in \pi$ there exists $m_{\alpha} \geq 0$ such that $s_{\alpha} \beta=\beta-m_{\alpha} \alpha$. On the other hand, $s_{\alpha} \beta=\beta-\sum_{\alpha^{\prime} \in \pi} c_{\alpha^{\prime}} a_{\alpha \alpha^{\prime}} \alpha$, that is, $A\left(c_{\alpha^{\prime}}\right)_{\alpha^{\prime} \in \pi} \geq 0$. Since $A$ is of indefinite type and $\left(c_{\alpha^{\prime}}\right)_{\alpha^{\prime} \in \pi}, A\left(c_{\alpha^{\prime}}\right)_{\alpha^{\prime} \in \pi}$ have only non-negative entries, we have a contradiction.

Let $V$ be a braided vector space of Cartan type with Cartan matrix $A$.

Lemma 3.7. If there exists a root $\gamma \in \Delta_{+}^{V}$ of $\mathcal{B}(V)$ such that $q_{\gamma, \gamma}=1$, then $\operatorname{GKdim} \mathcal{B}(V)=\infty$.

Proof. The Cartan matrix $A$ is not of finite type, since otherwise $q_{i i} \neq 1$ for all $i$ and hence $q_{\gamma, \gamma} \neq 1$ for all roots $\gamma$. If $A$ is of affine type, then $\operatorname{GKdim} \mathcal{B}(V)=\infty$ by Proposition 3.1. We assume then that $A$ is of indefinite type. By [H1], $s_{\alpha}\left(\boldsymbol{\Delta}_{+}^{V}-\{\alpha\}\right)=\boldsymbol{\Delta}_{+}^{V}-\{\alpha\}$ for all simple roots $\alpha$. Since $q_{w \gamma, w \gamma}=q_{\gamma, \gamma}=1$ for all $w \in W$, all root vectors of degree $w \gamma$ with $w \in W$ have infinite height. By Lemma [3.6, $W \gamma$ is infinite. Thus $\mathcal{B}(V)$ has a restricted homogeneous PBW basis containing infinitely many PBW generators having infinite height. The claim follows by Lemma 2.3 .

3.3. Braided coideal subalgebras. Just in this Subsection, the field $\mathbb{k}$ is arbitrary. Let $H$ be a Hopf algebra with bijective antipode.

Proposition 3.8. [GH, Prop. 2.1]. Let $B$ be a bialgebra in ${ }_{H}^{H} \mathcal{Y D}$, let $K$ be a subalgebra of $B$ and let $I$ be a subobject of $K$ in ${ }_{H}^{H} \mathcal{Y D}$, such that it is a coideal of $B$, an ideal of $K$ and

$$
\Delta(K) \subseteq K \otimes K+I \otimes B
$$

Then $K / I$ inherits a structure of bialgebra in ${ }_{H}^{H} \mathcal{Y D}$ from $B$.

By (3.2), $K$ is a right coideal subalgebra of $B$.

Proof. The existence of $\Delta$ is verified by usual chasing in the following commutative diagram:

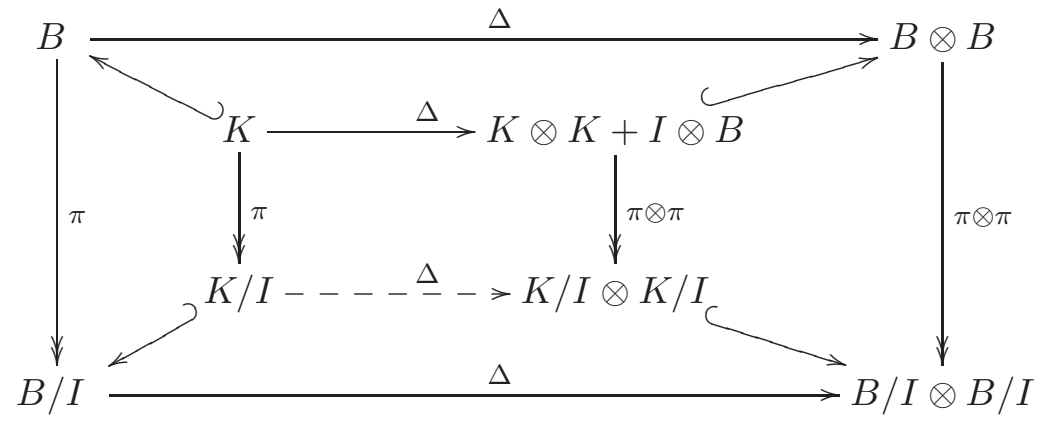

The associativity and compatibilities follow at once from those of $B$. 
We now apply Proposition 3.8 in the following context. Recall that $\alpha_{1}, \alpha_{2}$ is the canonical basis of $\mathbb{Z}^{2}$. Let $V=V_{1} \oplus V_{2}$ be a direct sum in ${ }_{H}^{H} \mathcal{Y} \mathcal{D}$. Then $\mathcal{B}(V)$ has a unique $\mathbb{N}_{0}^{2}$-grading (as a Hopf algebra in ${ }_{H}^{H} \mathcal{Y} \mathcal{D}$ )

$$
\mathcal{B}(V)=\bigoplus_{\alpha \in \mathbb{N}_{0}^{2}} \mathcal{B}^{\alpha}(V)
$$

such that $\operatorname{deg} V_{1}=\alpha_{1}$ and $\operatorname{deg} V_{2}=\alpha_{2}$. Let $r \in \mathbb{Q}_{\geq 0}$. We set

$$
\begin{aligned}
& B_{\geq r}=\bigoplus_{\substack{\alpha=a_{1} \alpha_{1}+a_{2} \alpha_{2} \in \mathbb{N}_{0}^{2}: \\
a_{1} \geq r a_{2}}} \mathcal{B}^{\alpha}(V), \quad B_{>r}=\bigoplus_{\substack{\alpha=a_{1} \alpha_{1}+a_{2} \alpha_{2} \in \mathbb{N}_{0}^{2}: \\
a_{1}>r a_{2}}} \mathcal{B}^{\alpha}(V), \\
& K_{\geq r}=\left\{x \in \mathcal{B}(V) \mid \Delta(x) \in B_{\geq r} \otimes \mathcal{B}(V)\right\}, \quad K_{>r}=K_{\geq r} \cap B_{>r} .
\end{aligned}
$$

Proposition 3.9. Let $r \in \mathbb{Q}$ with $r \geq 0$. Then the braided bialgebra structure of $\mathcal{B}(V)$ induces a braided Hopf algebra structure on $K_{\geq r} / K_{>r}$.

Proof. We claim that:

(i) $K_{\geq r} \subseteq B_{\geq r}$,

(ii) $K_{\geq r}$ is a subalgebra of $\mathcal{B}(V)$ in ${ }_{H}^{H} \mathcal{Y D}$,

(iii) $\Delta\left(K_{\geq r}\right) \subseteq K_{\geq r} \otimes K_{\geq r}+K_{>r} \otimes \mathcal{B}(V)$,

(iv) $K_{>r}$ is an ideal of $K_{\geq r}$ and a coideal of $\mathcal{B}(V)$ in ${ }_{H}^{H} \mathcal{Y D}$.

For (i), apply (id $\otimes \varepsilon$ ) to the inclusion defining $K_{\geq r}$. Now (ii) follows since the multiplication and the Yetter-Drinfeld structure of $\mathcal{B}(V)$ are $\mathbb{N}_{0}^{2}$-graded.

For (iii), note that $K_{\geq r}$ is a right coideal since $\Delta$ is coassociative. Using this fact and that $\Delta$ is $\mathbb{N}_{0}^{2}$-graded,

$$
\Delta\left(K_{\geq r}\right) \subseteq K_{\geq r} \otimes B_{\geq r}+K_{>r} \otimes B(V) .
$$

Indeed, let $x \in K_{\geq r}$ of degree $a_{1} \alpha_{1}+a_{2} \alpha_{2}$. We write $\Delta(x)=\sum_{i} y_{i} \otimes z_{i}$ with $y_{i}, z_{i} \mathbb{N}_{0}^{2}$-homogeneous. If $y_{i}, z_{i}$ have degree $b_{1} \alpha_{1}+b_{2} \alpha_{2}, c_{1} \alpha_{1}+c_{2} \alpha_{2}$, then either $b_{1}>r b_{2}$ so $y_{i} \otimes z_{i} \in K_{>r} \otimes B(V)$, or else $b_{1}=r b_{2}$, in which case $c_{1}=a_{1}-b_{1} \geq r a_{2}-r b_{2}=r c_{2}$ and $y_{i} \otimes z_{i} \in K_{\geq r} \otimes B_{\geq r}$. Now

$$
\begin{aligned}
& (\Delta \otimes \mathrm{id}) \Delta\left(K_{\geq r}\right) \subseteq(\Delta \otimes \mathrm{id})\left(K_{\geq r} \otimes B_{\geq r}+K_{>r} \otimes \mathcal{B}(V)\right) \\
& \quad \subseteq K_{\geq r} \otimes K_{\geq r} \otimes B_{\geq r}+K_{\geq r} \otimes K_{>r} \otimes \mathcal{B}(V)+K_{>r} \otimes \mathcal{B}(V) \otimes \mathcal{B}(V) .
\end{aligned}
$$

We apply (id $\otimes \mathrm{id} \otimes \varepsilon$ ) to the previous inclusion and get (iii).

Finally we prove (iv); Let $x \in K_{>r}, y \in K_{\geq r}$, we may assume they are homogeneous of degrees $\alpha=a_{1} \alpha_{1}+a_{2} \alpha_{2}, \beta=b_{1} \alpha_{1}+b_{2} \alpha_{2}$, so $a_{1}>r a_{2}$, $b_{1} \geq r b_{2}$. Hence $x y \in K_{\geq r}$ by (ii), and $x y$ has degree $\alpha+\beta=\left(a_{1}+b_{1}\right) \alpha_{1}+$ $\left(a_{2}+b_{2}\right) \alpha_{2}$ with $a_{1}+b_{1}>r\left(a_{2}+b_{2}\right)$, so $x y \in K_{>r}$. Analogously, $y x \in K_{>r}$. Thus $K_{>r}$ is an ideal of $K_{\geq r}$. Now $\Delta(x) \in K_{\geq r} \otimes K_{\geq r}+K_{>r} \otimes \mathcal{B}(V)$ by (iii), For each $u \otimes v \in K_{\geq r} \otimes K_{\geq r}$ appearing in $\Delta(x), u, v$ homogeneous of degrees $c_{1} \alpha_{1}+c_{2} \alpha_{2}, d_{1} \alpha_{1}+d_{2} \alpha_{2}$, we have that $c_{1} \geq r c_{2}, d_{1} \geq r d_{2}$. As $\Delta$ is $\mathbb{N}_{0^{-}}^{2}$ graded, $c_{1}+d_{1}=a_{1}, c_{2}+d_{2}=a_{2}$, so either $c_{1}>r c_{2}$ or else $d_{1}>r d_{2}$; in other words, either $u \in K_{>r}$ or else $v \in K_{>r}$, so $\Delta(x) \in K_{\geq_{r}} \otimes K_{>r}+K_{>r} \otimes \mathcal{B}(V)$. Thus $K_{>r}$ is a coideal of $\mathcal{B}(V)$ in ${ }_{H}^{H} \mathcal{Y D}$. 
Hence we may apply Proposition 3.8 and $K_{\geq r} / K_{>r}$ is a bialgebra in ${ }_{H}^{H} \mathcal{Y D}$. Since $\mathcal{B}(V)$ is $\mathbb{N}_{0}$-graded and connected and $\Delta$ is $\mathbb{N}_{0}$-graded, $K_{\geq r}$ and $K_{>r}$ are also $\mathbb{N}_{0}$-graded. Therefore $K_{\geq r} / K_{>r}$ is $\mathbb{N}_{0}$-graded and connected, so it is a Hopf algebra in ${ }_{H}^{H} \mathcal{Y} \mathcal{D}$ by [Mo, 5.2.10].

\section{RANK 2}

This Section contains a proof of the following result:

Theorem 4.1. Let $V$ be a braided vector space of diagonal type and dimension 2 such that $\mathrm{GKdim} \mathcal{B}(V)<\infty$. Then its generalized root system is finite.

Let $Z$ be a vector space and $\left(z_{i}\right)_{i \in I}$ a family of vectors in $Z$. Then $\left\langle z_{i}: i \in I\right\rangle$ denotes the subspace of $Z$ generated by the $z_{i}$ 's.

4.1. Lemmas for $\theta=2$. We establish some properties needed in the proof of Theorem 4.1. We keep the notation in 92.3 . we assume that $\theta=2$ and set as above $y_{k}=\left(\operatorname{ad} x_{1}\right)^{k} x_{2} \in \mathcal{B}(V)$. We also set:

$$
\beta_{m}=m \alpha_{1}+\alpha_{2}, \quad p_{m}=q_{\beta_{m} \beta_{m}}=q_{11}^{m^{2}} q_{12}^{m} q_{21}^{m} q_{22}, \quad m \in \mathbb{N}_{0} .
$$

4.1.1. On the powers of the $y_{n}$ 's.

Lemma 4.2. Let $r, s, t \in \mathbb{N}_{0}, r \leq s$. Then

$$
\partial_{1}^{t} \partial_{2}\left(y_{r} y_{s}\right)= \begin{cases}0, & s<t ; \\ \mu_{s}(s)_{q_{11}}(s-1)_{q_{11}} \ldots(s-t+1)_{q_{11}} y_{r} x_{1}^{s-t}, & r<t \leq s .\end{cases}
$$

Proof. By (2.3) we have that

$$
\partial_{2}\left(y_{r} y_{s}\right)=\mu_{r} q_{21}^{s} q_{22} x_{1}^{r} y_{s}+\mu_{s} y_{r} x_{1}^{s} \quad \text { for all } r, s \in \mathbb{N}_{0} .
$$

Using (2.3) again, if $t>s$, then $\partial_{1}^{t} \partial_{2}\left(y_{r} y_{s}\right)=0$, and if $r<t \leq s$,

$$
\partial_{1}^{t} \partial_{2}\left(y_{r} y_{s}\right)=\partial_{1}^{t}\left(\mu_{s} y_{r} x_{1}^{s}\right)=\mu_{s}(s)_{q_{11}}(s-1)_{q_{11}} \ldots(s-t+1)_{q_{11}} y_{r} x_{1}^{s-t}
$$

by induction on $t$.

Lemma 4.3. Let $l \in \mathbb{N}_{0}$. Assume that

$$
x_{1}^{2 l+1} \neq 0, \quad x_{2}^{2} \neq 0, \quad y_{l+1} \neq 0, \quad y_{l+1} y_{l} \in\left\langle y_{r} y_{s}: 0 \leq r \leq s\right\rangle .
$$

Then $q_{11}^{l(l+1)}\left(\widetilde{q}_{12}\right)^{l+1} q_{22}=1$ and

$$
y_{l+1} y_{l}=q_{11}^{l(l+1)} q_{12}^{l+1} q_{21}^{l} q_{22} y_{l} y_{l+1} .
$$

Proof. The last assumption of the lemma implies that

$$
y_{l+1} y_{l} \in\left\langle y_{r} y_{s}: 0 \leq r \leq s, r+s=2 l+1\right\rangle .
$$

Using Lemma 4.2 we have that

$$
\partial_{1}^{l+2} \partial_{2}\left(y_{l+1} y_{l}\right)=0, \quad \partial_{1}^{l+2} \partial_{2}\left(y_{r} y_{s}\right) \in \mathbb{k} y_{r} x_{1}^{s-l-2}-0,
$$


for all $r, s \in \mathbb{N}_{0}$ with $r+s=2 l+1, s \geq l+2$ such that $y_{s} \neq 0$. Thus there exists $\lambda \in \mathbb{k}$ such that $y_{l+1} y_{l}=\lambda y_{l} y_{l+1}$. Now we apply $\partial_{1}^{l+1} \partial_{2}$ to this equation. Using that $\partial_{1}^{l+1}\left(x_{1}^{l}\right)=0, \partial_{1}^{l+1}\left(x_{1}^{l+1}\right)=(l+1)_{q_{11}}^{!} \neq 0$ we obtain

$$
\mu_{l+1} q_{21}^{l} q_{22}\left(q_{11}^{l} q_{12}\right)^{l+1} y_{l}=\lambda \mu_{l+1} y_{l} .
$$

Thus $\lambda=q_{11}^{l(l+1)} q_{12}^{l+1} q_{21}^{l} q_{22}$, since $y_{l+1} \neq 0$. Finally we apply $\partial_{2}^{2}$ to (4.3). Since $\mu_{l+1} \neq 0$ and $x_{1}^{2 l+1} \neq 0$, we obtain that

$$
q_{21}^{l} q_{22}+q_{21}^{l}=q_{11}^{l(l+1)} q_{12}^{l+1} q_{21}^{l} q_{22}\left(q_{21}^{l+1} q_{22}+q_{21}^{l+1}\right) .
$$

As $x_{2}^{2} \neq 0$, we have that $q_{22} \neq-1$. Hence $q_{11}^{l(l+1)}\left(\widetilde{q}_{12}\right)^{l+1} q_{22}=1$.

Lemma 4.4. Let $l \in \mathbb{N}$. Assume that $y_{l}^{2} \in\left\langle y_{r} y_{s}: 0 \leq r<s\right\rangle$ and that $y_{l} \neq 0$. Then $y_{l+1}=0$ and $q_{11}^{l^{2}}\left(\widetilde{q}_{12}\right)^{l} q_{22}=-1$.

Proof. As $\mathcal{B}(V)$ is $\mathbb{N}_{0}$-graded, the last assumption says that

$$
y_{l}^{2} \in\left\langle y_{r} y_{s}: 0 \leq r<s, r+s=2 l\right\rangle .
$$

Using Lemma 4.2 we have that

$$
\partial_{1}^{l+1} \partial_{2}\left(y_{l}^{2}\right)=0, \quad \partial_{1}^{l+1} \partial_{2}\left(y_{r} y_{s}\right) \in \mathbb{k} y_{r} x_{1}^{s-l-1}-0,
$$

for all $r, s \in \mathbb{N}_{0}$ with $r<l, r+s=2 l, y_{s} \neq 0$. Thus $y_{l}^{2}=0$. Since $l>0$,

$$
0=\partial_{1}^{l-1} \partial_{2}\left(y_{l}^{2}\right)=a_{1} x_{1} y_{l}+a_{2} y_{l} x_{1}
$$

for some $a_{1}, a_{2} \in \mathbb{k}-0$. Since $x_{1} y_{l}=y_{l+1}+q_{11}^{l} q_{12} y_{l} x_{1}$ and since $y_{l+1}$ and $y_{l} x_{1}$ are linearly independent whenever $y_{l+1}=0$, the latter equation implies that $y_{l+1}=0$. Therefore $x_{1} y_{l}=q_{11}^{l} q_{12} y_{l} x_{1}$ and we have that

$$
0=\partial_{2}\left(y_{l}^{2}\right)=\mu_{r}\left(q_{21}^{l} q_{22}\left(q_{11}^{l} q_{12}\right)^{l}+1\right) y_{l} x_{1}^{l} .
$$

This implies the last claim.

We fix $n>0$ and set $q=p_{n}$, cf. (4.1). Assume that $q \in \mathbb{G}_{N}^{\prime}$ for some $N \geq 2$. In the next few Lemmas we prepare a condition for $y_{n}^{N}$ being a root vector. We start with some computations with $q$-numbers. Recall that

$$
\left(t_{1}+t_{2}\right)_{q}=\left(t_{1}\right)_{q}+q^{t_{1}}\left(t_{2}\right)_{q}, \quad \text { for all } t_{1}, t_{2} \in \mathbb{N}_{0} .
$$

In particular, $(N)_{q}=0$ and $(N-t)_{q}=-q^{-t}(t)_{q}$ for all $0 \leq t \leq N$.

Lemma 4.5. Let $r \in\{0,1, \ldots, N-2\}$ and $t \in \mathbb{N}_{0}$. Then

$$
\sum_{l=0}^{t} q^{l}(l+1)_{q}(l+2)_{q} \cdots(l+r)_{q}=\frac{(t+1)_{q}(t+2)_{q} \cdots(t+r+1)_{q}}{(r+1)_{q}} .
$$

Proof. Note that $(r+1)_{q} \neq 0$ since $1 \leq r+1<N$. We proceed by induction on $t$. For $t=0$ the claim is trivial. For any $t \geq 0$, for which the claim holds, we obtain that

$$
\sum_{l=0}^{t+1} q^{l}(l+1)_{q}(l+2)_{q} \cdots(l+r)_{q}
$$




$$
\begin{aligned}
& =\sum_{l=0}^{t} q^{l}(l+1)_{q}(l+2)_{q} \cdots(l+r)_{q}+q^{t+1}(t+2)_{q} \cdots(t+r+1)_{q} \\
& =\frac{(t+1)_{q}(t+2)_{q} \cdots(t+r+1)_{q}}{(r+1)_{q}}+q^{t+1}(t+2)_{q}(t+3)_{q} \cdots(t+r+1)_{q} \\
& =\frac{(t+1)_{q}+q^{t+1}(r+1)_{q}}{(r+1)_{q}}(t+2)_{q}(t+3)_{q} \cdots(t+r+1)_{q} \\
& =\frac{(t+2)_{q}(t+3)_{q} \cdots(t+r+1)_{q}(t+r+2)_{q}}{(r+1)_{q}}
\end{aligned}
$$

This proves the claim.

For any $t \in\{0,1, \ldots, N-2\}$ let

$$
Y(t)=\sum_{j=0}^{t}\left(q_{11}^{n} q_{12}\right)^{-j} \frac{(N-t-1+j)_{q}^{!}}{(j)_{q}^{!}} y_{n}^{t-j} y_{n+1} y_{n}^{j} .
$$

In particular, $Y(0)=(N-1)_{q}^{!} y_{n+1}$.

Lemma 4.6. $\partial_{1}^{n-1} \partial_{2}\left(y_{n}^{N}\right)=-\mu_{n}(n)_{q_{11}}^{!}\left(q_{11}^{n} q_{12}\right)^{-1} Y(N-2)$.

Proof. First we obtain that

$$
\partial_{2}\left(y_{n}^{N}\right)=\sum_{l=0}^{N-1} y_{n}^{N-1-l} \partial_{2}\left(y_{n}\right) \alpha_{2} \cdot y_{n}^{l}=\mu_{n} \sum_{l=0}^{N-1}\left(q_{21}^{n} q_{22}\right)^{l} y_{n}^{N-1-l} x_{1}^{n} y_{n}^{l} .
$$

Since $\partial_{1}\left(y_{n}\right)=0$, we have that

$$
\partial_{1}^{n-1} \partial_{2}\left(y_{n}^{N}\right)=\mu_{n}(n)_{q_{11}}^{!} \sum_{l=0}^{N-1}\left(q_{11}^{n(n-1)} q_{12}^{n-1} q_{21}^{n} q_{22}\right)^{l} y_{n}^{N-1-l} x_{1} y_{n}^{l} .
$$

The equation $y_{n+1}=x_{1} y_{n}-q_{11}^{n} q_{12} y_{n} x_{1}$ implies that

$$
x_{1} y_{n}^{l}=q_{11}^{n l} q_{12}^{l} y_{n}^{l} x_{1}+\sum_{j=0}^{l-1}\left(q_{11}^{n} q_{12}\right)^{l-1-j} y_{n}^{l-1-j} y_{n+1} y_{n}^{j} .
$$

Therefore

$$
\begin{aligned}
\partial_{1}^{n-1} \partial_{2}\left(y_{n}^{N}\right)= & \mu_{n}(n)_{q_{11}}^{!} \sum_{l=0}^{N-1}\left(q_{11}^{n(n-1)} q_{12}^{n-1} q_{21}^{n} q_{22}\right)^{l} y_{n}^{N-1-l} . \\
& \left(q_{11}^{n l} q_{12}^{l} y_{n}^{l} x_{1}+\sum_{j=0}^{l-1}\left(q_{11}^{n} q_{12}\right)^{l-1-j} y_{n}^{l-1-j} y_{n+1} y_{n}^{j}\right) \\
= & \mu_{n}(n)_{q_{11}}^{!}\left(\sum_{l=0}^{N-1} q^{l} y_{n}^{N-1} x_{1}\right. \\
& \left.+\left(q_{11}^{n} q_{12}\right)^{-1} \sum_{j=0}^{N-2}\left(\sum_{l=j+1}^{N-1} q^{l}\right)\left(q_{11}^{n} q_{12}\right)^{-j} y_{n}^{N-2-j} y_{n+1} y_{n}^{j}\right)
\end{aligned}
$$


Hence the Lemma follows since $\sum_{l=0}^{N-1} q^{l}=0$ and $\sum_{l=j+1}^{N-1} q^{l}=-(j+1)_{q}$.

For any $1 \leq t \leq N-1$ let

$$
d_{t}=1-q^{t+1} q_{11}^{2 n} \widetilde{q}_{12}+\frac{q^{t}\left(1-q_{11}^{n} \widetilde{q}_{12}\right)(n+1)_{q_{11}}}{(t)_{q}} .
$$

Observe that $d_{t}$ depends on $n$.

Lemma 4.7. Let $t \in\{1,2, \ldots, N-1\}$. Then

$$
\partial_{1}^{n} \partial_{2}(Y(t))=\mu_{n}(n)_{q_{11}}^{!}\left(q_{11}^{n} q_{12}\right)^{-1} d_{t} Y(t-1) .
$$

Proof. Similarly to the calculation in Lemma 4.6 we have that

$$
\begin{aligned}
\partial_{1}^{n} \partial_{2}(Y(t))= & \sum_{l=0}^{t}\left(q_{11}^{n} q_{12}\right)^{-l}\left(\prod_{i=1}^{N-t-1}(l+i)_{q}\right) . \\
& \left(\partial_{1}^{n} \partial_{2}\left(y_{n}\right)(t-l)_{q} q^{l+1} q_{11}^{n} q_{21} y_{n}^{t-1-l} y_{n+1} y_{n}^{l}\right. \\
& +\partial_{1}^{n} \partial_{2}\left(y_{n}\right)\left(1-q_{11}^{n} \widetilde{q}_{12}\right)(n+1)_{q_{11}} q^{l} y_{n}^{t-l} x_{1} y_{n}^{l} \\
& \left.+\partial_{1}^{n} \partial_{2}\left(y_{n}\right)(l)_{q} y_{n}^{t-l} y_{n+1} y_{n}^{l-1}\right) .
\end{aligned}
$$

Using (4.5) this implies that

$$
\begin{aligned}
& \partial_{1}^{n} \partial_{2}(Y(t))=\partial_{1}^{n} \partial_{2}\left(y_{n}\right) \sum_{j=0}^{t-1}\left(q_{11}^{n} q_{12}\right)^{-j}\left(\prod_{i=1}^{N-t-1}(j+i)_{q}\right) \\
& \left(-q^{t-j}\right)(N-t+j)_{q} q^{j+1} q_{11}^{n} q_{21} y_{n}^{t-1-j} y_{n+1} y_{n}^{j} \\
& +\partial_{1}^{n} \partial_{2}\left(y_{n}\right)\left(1-q_{11}^{n} \widetilde{q}_{12}\right)(n+1)_{q_{11}} \sum_{l=0}^{t}\left(q_{11}^{n} q_{12}\right)^{-l}\left(\prod_{i=1}^{N-t-1}(l+i)_{q}\right) q^{l} \\
& \quad\left(q_{11}^{n l} q_{12}^{l} y_{n}^{t} x_{1}+\sum_{j=0}^{l-1}\left(q_{11}^{n} q_{12}\right)^{l-1-j} y_{n}^{t-1-j} y_{n+1} y_{n}^{j}\right) \\
& +\partial_{1}^{n} \partial_{2}\left(y_{n}\right) \sum_{j=0}^{t-1}\left(q_{11}^{n} q_{12}\right)^{-j-1}\left(\prod_{i=1}^{N-t-1}(j+1+i)_{q}\right)(j+1)_{q} y_{n}^{t-1-j} y_{n+1} y_{n}^{j}
\end{aligned}
$$

Lemma 4.5 tells that

$$
\sum_{l=0}^{t} q^{l} \prod_{i=1}^{N-t-1}(l+i)_{q}=\prod_{i=1}^{N-t}(t+i)_{q} /(N-t)_{q}=0
$$

since $1<t<N$. Therefore the terms $y_{n}^{t} x_{1}$ disappear in the above expression for $\partial_{1}^{n} \partial_{2}(Y(t))$. Moreover,

$$
\sum_{l=0}^{t} \sum_{j=0}^{l-1}\left(\prod_{i=1}^{N-t-1}(l+i)_{q}\right) q^{l}\left(q_{11}^{n} q_{12}\right)^{-1-j}
$$




$$
\begin{aligned}
= & \sum_{j=0}^{t-1}\left(q_{11}^{n} q_{12}\right)^{-1-j} \sum_{l=j+1}^{t} q^{l}\left(\prod_{i=1}^{N-t-1}(l+i)_{q}\right) \\
= & \sum_{j=0}^{t-1}\left(q_{11}^{n} q_{12}\right)^{-1-j}\left(\sum_{l=0}^{t} q^{l}\left(\prod_{i=1}^{N-t-1}(l+i)_{q}\right)-\sum_{l=0}^{j} q^{l}\left(\prod_{i=1}^{N-t-1}(l+i)_{q}\right)\right) \\
= & \sum_{j=0}^{t-1}\left(q_{11}^{n} q_{12}\right)^{-1-j}\left(\prod_{i=1}^{N-t}(t+i)_{q}-\prod_{i=1}^{N-t}(j+i)_{q}\right) /(N-t)_{q} \\
= & -\sum_{j=0}^{t-1}\left(q_{11}^{n} q_{12}\right)^{-1-j}\left(\prod_{i=1}^{N-t}(j+i)_{q}\right) /(N-t)_{q} .
\end{aligned}
$$

Therefore,

$$
\begin{aligned}
\partial_{1}^{n} \partial_{2}(Y(t))= & \partial_{1}^{n} \partial_{2}\left(y_{n}\right)\left(q_{11}^{n} q_{12}\right)^{-1} \sum_{j=0}^{t-1}\left(q_{11}^{n} q_{12}\right)^{-j}\left(\prod_{i=1}^{N-t}(j+i)_{q}\right) \\
& \left(-q^{t+1} q_{11}^{2 n} \widetilde{q}_{12}-\frac{\left(1-q_{11}^{n} \widetilde{q}_{12}\right)(n+1)_{q_{11}}}{(N-t)_{q}}+1\right) y_{n}^{t-1-j} y_{n+1} y_{n}^{j}
\end{aligned}
$$

Thus the claim follows from this equality and $(N-t)_{q}=-q^{-t}(t)_{q}$.

Proposition 4.8. Assume that $y_{n}^{N}=0$ and $y_{n+1} \neq 0$. Then $d_{t}=0$ for some $1 \leq t \leq N-2$, see (4.6).

Proof. Lemma 4.6 implies that $Y(N-2)=0$. Thus

$$
\left(\partial_{1}^{n} \partial_{2}\right)^{N-2}(Y(N-2))=0 .
$$

By Lemma 4.7 either $d_{N-2}=0$ or else $Y(N-3)=0$. Recursively, if $Y(t)=0$, then either $d_{t}=0$ or else $Y(t-1)=0$, for each $1 \leq t \leq N-2$. Since $Y(0)=(N-1)_{q}^{!} y_{n+1} \neq 0$, necessarily $d_{t}=0$ for some $1 \leq t \leq N-2$.

Lemma 4.9. If $y_{n}^{N} \neq 0$ then $N \beta_{n}$ is a root of $V$.

Proof. By the definition of roots of $\mathcal{B}(V)$, either $\operatorname{deg} y_{n}^{N}=N\left(n \alpha_{1}+\alpha_{2}\right)$ is a root of $V$ or $y_{n}^{N}$ can be expressed as a linear combination of products $\ell_{1}^{m_{1}} \cdots \ell_{k}^{m_{k}}$ as in (2.5), where each $\ell_{i}$ corresponds either to a Lyndon word $l_{i}$ greater than $x_{1}^{n} x_{2}$, or else to a power of this kind of letters.

Assume the last case holds. Then each $l_{i}$ starts with $1^{k} 2$, where $k \leq n$, and ends with 2. Since $l_{i}$ is a Lyndon word, any end of $l_{i}$ is larger than $v$, and hence it contains no subword $1^{l} 2$ with $l>k$. Therefore $l_{i}=x_{1}^{k_{1}} x_{2} \cdots x_{1}^{k_{r}} x_{2}$ with $k_{1}, \ldots, k_{r} \leq n$ and $k_{1}+\cdots+k_{r}<r n$. This implies that $\operatorname{deg} \ell_{1} \cdots \ell_{m} \neq$ $\operatorname{deg} y_{n}^{N}$ so $y_{n}^{N}=0$, a contradiction. Thus $y_{n}$ has infinite height and hence $N \beta_{n}$ is a root of $\mathcal{B}(V)$.

Lemma 4.10. Let $m \in \mathbb{N}_{0}$ with $y_{m} \neq 0$. Then $y_{m}^{2}=0$ if and only if $p_{m}=-1$ and $y_{m+1}=0$. 
Proof. If $y_{m}^{2}=0$, then Lemma 4.4 says that $y_{m+1}=0$ and $p_{m}=-1$.

Conversely, assume that $y_{m+1}=0, y_{m} \neq 0$, and $p_{m}=-1$. Then

$$
\begin{aligned}
\partial_{2}\left(y_{m}^{2}\right) & =\mu_{m}\left(y_{m} x_{1}^{m}+x_{1}^{m} q_{21}^{m} q_{22} y_{m}\right) \\
& =\mu_{m}\left(1+\left(q_{11}^{m} q_{12}\right)^{m} q_{21}^{m} q_{22}\right) y_{m} x_{1}^{m}=0 .
\end{aligned}
$$

Since $\partial_{1}\left(y_{m}\right)=0$, we conclude that $y_{m}^{2}=0$.

Lemma 4.11. Let $m \in \mathbb{N}_{0}$. Assume that $p_{m}=-1$ and $y_{m+1} \neq 0$. Then $2 \beta_{m}$ is a root of $V$ and $q_{2 \beta_{m} \beta_{m}}=1$.

Proof. As $y_{m+1} \neq 0$, Lemma 4.10 says that $y_{m}^{2} \neq 0$. Now Lemma 4.9 applies and $2 \beta_{m}$ is a root. Finally, $q_{2 \beta_{m}} 2 \beta_{m}=p_{m}^{4}=1$.

4.1.2. On the $w_{m}$ 's. We consider the following elements of $\mathcal{B}(V)$ :

$$
w_{m}=y_{m+2} y_{m}-q_{\beta_{m+2}, \beta_{m}} y_{m} y_{m+2}, \quad m \in \mathbb{N}_{0} \text {. }
$$

Notice that $w_{m}$ is $\mathbb{N}_{0}^{2}$-homogeneous of degree $2 \beta_{m+1}$.

Let $m \in \mathbb{N}_{0}$. Assume that $y_{m+2} \neq 0$ and $q_{\beta_{m+1} \beta_{m+1}} \neq-1$. Let

$$
\widetilde{w}_{m}=w_{m}-\frac{q_{\beta_{m+1} \beta_{m}}(m+2)_{q_{11}}\left(1-q_{11}^{m+1} \widetilde{q}_{12}\right)}{1+q_{\beta_{m+1} \beta_{m+1}}} y_{m+1}^{2} .
$$

Our next goal is to determine when $\widetilde{w}_{m}=0$.

Lemma 4.12. Let $m \in \mathbb{N}_{0}$. Assume that

$$
y_{m+2} \neq 0, \quad p_{m+1} \neq-1, \quad w_{k} \in \mathbb{k} y_{k+1}^{2}, \quad 0 \leq k<m .
$$

Then the following are equivalent:

(1) $w_{m} \in \mathbb{k} y_{m+1}^{2}$.

(2) $\widetilde{w}_{m}=0$

(3) $\partial_{1}^{m} \partial_{2}\left(\widetilde{w}_{m}\right)=0$.

(4) The following equation holds:

$$
0=\left(1-\frac{p_{m+1}}{q_{11}}\right)\left(1+\frac{p_{m+1}}{q_{11}}+\frac{p_{m}(m+2)_{q_{11}}^{!}\left(1-q_{11}^{m} \widetilde{q}_{12}\right)\left(1-q_{11}^{m+1} \widetilde{q}_{12}\right)}{(m)_{q_{11}}^{!}\left(1+q_{11}\right)\left(1+p_{m+1}\right)}\right)
$$

Proof. First we compute

$$
\begin{gathered}
\partial_{1}^{m} \partial_{2}\left(w_{m}\right)=\partial_{1}^{m}\left(\mu_{m+2} x_{1}^{m+2} q_{21}^{m} q_{22} y_{m}+y_{m+2} \mu_{m} x_{1}^{m}\right) \\
-q_{\beta_{m+2} \beta_{m}}\left(\mu_{m} x_{1}^{m} q_{21}^{m+2} q_{22} y_{m+2}+y_{m} \mu_{m+2} x_{1}^{m+2}\right) \\
=\mu_{m+2} q_{\beta_{m} \beta_{m}} \frac{(m+2)_{q_{11}}^{!}}{1+q_{11}}\left(x_{1}^{2} y_{m}-q_{11}^{2 m} q_{12}^{2} y_{m} x_{1}^{2}\right) \\
+\mu_{m}(m)_{q_{11}}^{!}\left(1-q_{\beta_{m+2} \beta_{m}} q_{\beta_{m} \beta_{m+2}}\right) y_{m+2} .
\end{gathered}
$$

Similarly, we compute

$$
\partial_{1}^{m} \partial_{2}\left(y_{m+1}^{2}\right)=\mu_{m+1}(m+1)_{q_{11}}^{!}\left(q_{\beta_{m} \beta_{m+1}} y_{m+2}+\left(1+p_{m+1}\right) y_{m+1} x_{1}\right),
$$

see also the calculation in the proof of Lemma 4.10. 
(1) $\Longrightarrow$ (2). Using the previous formulas one obtains quickly that

$$
\begin{aligned}
\partial_{1}^{m+1} \partial_{2}\left(w_{m}\right) & =\mu_{m+2} q_{\beta_{m+1} \beta_{m}}(m+2)_{q_{11}}^{!} y_{m+1}, \\
\partial_{1}^{m+1} \partial_{2}\left(y_{m+1}^{2}\right) & =\mu_{m+1}(m+1)_{q_{11}}\left(1+p_{m+1}\right) y_{m+1} .
\end{aligned}
$$

Since $y_{m+1} \neq 0$, this implies the claim.

(2) $\Longrightarrow$ (3). Trivial.

(3) $\Longleftrightarrow$ (41). We notice that

$$
x_{1}^{2} y_{m}-q_{11}^{2 m} q_{12}^{2} y_{m} x_{1}^{2}=y_{m+2}+\left(1+q_{11}\right) q_{11}^{m} q_{12} y_{m+1} x_{1} .
$$

Since $y_{m+2}$ and $y_{m+1} x_{1}$ are linearly independent in $\mathcal{B}(V)$, the formulas at the beginning of the proof imply that (3) is equivalent to

$$
\begin{aligned}
& \frac{p_{m}(m+1)_{q_{11}}(m+2)_{q_{11}}\left(1-q_{11}^{m} \widetilde{q}_{12}\right)\left(1-q_{11}^{m+1} \widetilde{q}_{12}\right)}{1+q_{11}}+1-q_{11}^{-2} p_{m+1}^{2} \\
& -\frac{q_{11}^{-1} p_{m} p_{m+1}(m+1)_{q_{11}}(m+2)_{q_{11}}\left(1-q_{11}^{m} \widetilde{q}_{12}\right)\left(1-q_{11}^{m+1} \widetilde{q}_{12}\right)}{1+p_{m+1}}=0 .
\end{aligned}
$$

This gives the equivalence between (3) and (4).

(3) $\Longrightarrow$ (11). We prove by induction on $k$ that

$$
\partial_{1}^{m-k} \partial_{2}\left(\widetilde{w}_{m}\right)=0 \quad \text { for all } 0 \leq k \leq m .
$$

If so, then $\partial_{i}\left(\widetilde{w}_{m}\right)=0$ for $i=1,2$ and then $\widetilde{w}_{m}=0$.

For $k=0$, (4.9) holds by assumption. Now let $k>0$ and assume the statement holds for $j<k$. Notice that

$$
\begin{aligned}
\operatorname{ker} \partial_{1} \cap \mathcal{B}^{(m+k+2) \alpha_{1}+\alpha_{2}}=\mathbb{k} y_{m+k+2} \\
\quad \Longrightarrow \partial_{1}^{m-k} \partial_{2}\left(\widetilde{w}_{m}\right)=b y_{m+k+2} \text { for some } b \in \mathbb{k} .
\end{aligned}
$$

We may assume that $y_{m+k+2} \neq 0$, otherwise the induction step holds.

Let $\iota: V \rightarrow V^{*}$ be the linear isomorphism with $x_{i} \mapsto \partial_{i}$ for $i \in \mathbb{I}$. Then $\iota$ is an isomorphism of braided vector spaces and hence induces an isomorphism between the Hopf algebras $\mathcal{B}(V)$ and $\mathcal{B}\left(V^{*}\right)$ in ${ }_{H}^{H} \mathcal{Y} \mathcal{D}$. Notice that

$$
\iota\left(y_{m-j}\right)\left(\widetilde{w}_{m}\right)=\partial_{1}^{m-j} \partial_{2}\left(\widetilde{w}_{m}\right)=0 \quad \text { for all } 0 \leq j<k,
$$

since $y_{j}=x_{1}^{j} x_{2}+$ terms ending in $x_{1}$ and $\partial_{1}\left(\widetilde{w}_{m}\right)=0$. Hence

$$
\begin{aligned}
\iota\left(w_{m-k}\right)\left(\widetilde{w}_{m}\right) & =\iota\left(y_{m-k+2} y_{m-k}-q_{\beta_{m-k+2}, \beta_{m-k}} y_{m-k} y_{m-k+2}\right)\left(\widetilde{w}_{m}\right) \\
& =\iota\left(y_{m-k+2}\right) \iota\left(y_{m-k}\right)\left(\widetilde{w}_{m}\right)=b \iota\left(y_{m-k+2}\right)\left(y_{m+k+2}\right) \\
& =b \partial_{1}^{m-k+2} \partial_{2}\left(y_{m+k+2}\right)=b \mu_{m+k+2} \frac{(m+k+2)_{q_{11}}}{(m-k+2)_{q_{11}}} .
\end{aligned}
$$

On the other hand, using that $w_{m-k}=a_{m-k} y_{m-k+1}^{2}$ for some $a_{m-k} \in \mathbb{k}$ and the inductive hypothesis,

$$
\iota\left(w_{m-k}\right)\left(\widetilde{w}_{m}\right)=a_{m-k} \iota\left(y_{m-k+1}^{2}\right)\left(\widetilde{w}_{m}\right)=a_{m-k} \iota\left(y_{m-k+1}\right) \partial_{1}^{m-k+1} \partial_{2}\left(\widetilde{w}_{m}\right)=0 .
$$

Hence $b=0$, which completes the inductive step. 
Remark 4.13. In the following cases, the right hand side of the equation in Lemma 4.12 (4) is equal to the following:

(i) $m=0:\left(1+q_{22}\right)\left(1-\widetilde{q}_{12} q_{22}\right)\left(1+q_{11} \widetilde{q}_{12}^{2} q_{22}\right)\left(1+q_{11} \widetilde{q}_{12} q_{22}\right)^{-1}$.

(ii) $m=1, q_{22}=-1$ :

$$
\left(1+q_{11}^{3} \widetilde{q}_{12}^{2}\right)\left(1-q_{11}^{3} \widetilde{q}_{12}\right)(3)_{-q_{11} \widetilde{q}_{12}}\left(1+q_{11}^{2} \widetilde{q}_{12}\right)^{-1} .
$$

(iii) $m=1, \widetilde{q}_{12} q_{22}=1$ :

$$
\left(1-q_{11}^{3} \widetilde{q}_{12}\right)(4)_{q_{11}}(3)_{-q_{11}^{2} \widetilde{q}_{12}}\left(1+q_{11}^{4} \widetilde{q}_{12}\right)^{-1} .
$$

(iv) $m=1, q_{11} \widetilde{q}_{12}^{2} q_{22}=-1$ :

$$
\left(1+q_{11}^{2}\right)\left(1-\widetilde{q}_{12}^{-1}\right)\left(1-q_{11}^{3} \widetilde{q}_{12}\right)\left(1-q_{11}\right)^{-1} .
$$

(v) $m=2, q_{22}=-1$ :

$$
\begin{aligned}
& \left(1+q_{11}^{8} \widetilde{q}_{12}^{3}\right)\left(1-q_{11}^{4} \widetilde{q}_{12}\right)(3)_{q_{11}^{3}}^{-1} \widetilde{q}_{12} \cdot \\
& \quad\left(q_{11}^{10} \widetilde{q}_{12}^{4}+\left(q_{11}^{7}+q_{11}^{6}\right) \widetilde{q}_{12}^{3}-(3)_{q_{11}} q_{11}^{4} \widetilde{q}_{12}^{2}+\left(q_{11}^{4}+q_{11}^{3}\right) \widetilde{q}_{12}+1\right) .
\end{aligned}
$$

(vi) $m=2, \widetilde{q}_{12} q_{22}=1, q_{11}^{2}=-1: 1-\widetilde{q}_{12}^{4}$.

(vii) $m=2, \widetilde{q}_{12} q_{22}=1,(3)_{-q_{11}^{2} \widetilde{q}_{12}}=0$ :

$$
\left(1+q_{11}^{4} \widetilde{q}_{12}\right)\left(1-q_{11}^{4} \widetilde{q}_{12}\right)\left(1-q_{11}^{5} \widetilde{q}_{12}\right)(5)_{q_{11}}(3)_{-q_{11}}\left(1+q_{11}^{9} \widetilde{q}_{12}^{2}\right)^{-1} .
$$

Recall the definition of $\widetilde{w}_{m}, m \in \mathbb{N}_{0}$, given in (4.8). We study in the next Lemmas when $\widetilde{w}_{m} \neq 0$ for small values of $m$.

Lemma 4.14. Assume that $y_{2} \neq 0, p_{1} \neq-1$. Then $\widetilde{w}_{0}=0$ if and only if

$$
\left(\widetilde{q}_{12} q_{22}-1\right)\left(q_{22}+1\right)\left(q_{11} \widetilde{q}_{12}^{2} q_{22}+1\right)=0 .
$$

Proof. The claim follows by Lemma 4.12 and Remark 4.13 (i).

Next we give conditions on the matrix $\mathbf{q}$ which are equivalent to the equation $\widetilde{w}_{1}=0$.

Lemma 4.15. Assume that $y_{3} \neq 0, p_{2} \neq-1$.

(a) If $q_{22}=-1$, then $\widetilde{w}_{1}=0$ if and only if

$$
\left(1-q_{11}^{3} \widetilde{q}_{12}\right)\left(q_{11}^{3} \widetilde{q}_{12}^{2}+1\right)(3)_{-q_{11} \widetilde{q}_{12}}=0 .
$$

(b) If $\widetilde{q}_{12} q_{22}=1$, then $\widetilde{w}_{1}=0$ if and only if

$$
\left(1-q_{11}^{3} \widetilde{q}_{12}\right)\left(q_{11}^{2}+1\right)(3)_{-q_{11}^{2} \widetilde{q}_{12}}=0 .
$$

(c) If $q_{11} \widetilde{q}_{12}^{2} q_{22}=-1$, then $\widetilde{w}_{1}=0$ if and only if $\left(1-q_{11}^{3} \widetilde{q}_{12}\right)\left(q_{11}^{2}+1\right)=0$.

Proof. As we assume $y_{3} \neq 0$, we have that $(3)_{q_{11}}^{!} \mu_{3} \neq 0$. That is,

$$
q_{11} \notin \mathbb{G}_{2} \cup \mathbb{G}_{3}, \quad \quad q_{11}^{k} \widetilde{q}_{12} \neq 1, \quad k=0,1,2 .
$$

Assume that $q_{22}=-1$, so we have that $p_{1}=-q_{11} \widetilde{q}_{12} \neq-1$. Hence $\widetilde{w}_{0}=0$ by Lemma 4.14. Now (a) follows by Lemma 4.12 and Remark 4.13 (ii). 
To prove (b), we assume that $\widetilde{q}_{12} q_{22}=1$. Again we have that $p_{1}=q_{11} \neq$ -1 , so $\widetilde{w}_{0}=0$ by Lemma 4.14. Now we can apply Lemma 4.12 and Remark 4.13 (iii) and the claim follows.

Finally, if $q_{11} \widetilde{q}_{12}^{2} q_{22}=-1$, then $p_{1}=-\widetilde{q}_{12}^{-1} \neq-1$, so $\widetilde{w}_{0}=0$ by Lemma 4.14. Hence (c) follows by Lemma 4.12 and Remark 4.13 (iv).

4.2. Proof of Theorem 4.1. First we extend Lemma 3.7 to any braided vector space of diagonal type and dimension two.

Proposition 4.16. Assume that $V$ is of dimension two and $\widetilde{q}_{12} \neq 1$. If there is a root $\gamma$ of $V$ such that $q_{\gamma \gamma}=1$, then $\operatorname{GKdim} \mathcal{B}(V)=\infty$.

Proof. If $V$ does not admit all reflections, then $\operatorname{GKdim} \mathcal{B}(V)=\infty$ by Remark 2.5.

Now we assume that $V$ admits all reflections. If $V$ is generic, then $V$ is of Cartan type. As $\gamma$ cannot be a real root since $q_{\gamma \gamma}=1, V$ is not of finite type. Then $\operatorname{GKdim} \mathcal{B}(V)=\infty$ by Remark 3.2 (3).

If $V$ is semigeneric, then $G K \operatorname{dim} \mathcal{B}(V)=\infty$. Indeed if we suppose that $\operatorname{GKdim} \mathcal{B}(V)<\infty$, then all roots $\beta$ satisfy $q_{\beta} \neq 1$ since the root system is finite by Corollary [3.5, and this gives a contradiction.

Finally, if $V$ is of torsion class, then the set $\mathcal{X}$ is finite by Remark 3.2 (2). If the orbit of $\gamma$ is infinite, then $\operatorname{GKdim} \mathcal{B}(V)=\infty$ since there are infinitely many roots $\delta$ of $V$ with $q_{\delta, \delta}=1$. Now assume that $\gamma$ has finite orbit. Let $s_{1}, s_{2}$ be the simple reflections corresponding to $V$, cf. (2.8). Then there exists $k>0$ such that $\left(s_{1} s_{2}\right)^{k}(\gamma)=\gamma$ and $\left(\mathcal{R}^{1} \mathcal{R}^{2}\right)^{k}(V)=V$. Thus 1 is an eigenvalue of $\left(s_{1} s_{2}\right)^{k} \in \operatorname{Aut}\left(\mathbb{Z}^{2}\right)$. Since $\operatorname{det}\left(s_{1} s_{2}\right)=1$, the other eigenvalue is also 1. Thus either $\left(s_{1} s_{2}\right)^{k}=\mathrm{id}$, or else $\left(s_{1} s_{2}\right)^{k}$ is a shear mapping. The first case implies that the set of real roots is finite, hence the Weyl groupoid is finite by [CH], and consequently all roots $\delta$ of $V$ are real [CH], a contradiction.

In the second case there exist $c_{i} \in \mathbb{Z}$ such that $\left(s_{1} s_{2}\right)^{k}\left(\alpha_{i}\right)=\alpha_{i}+c_{i} \gamma$ for $i=1,2$. Hence, each $\beta_{n}:=\left(s_{1} s_{2}\right)^{n k}\left(\alpha_{i}\right)=\alpha_{i}+c_{i} n \gamma$ is a real root for $n \in \mathbb{N}$ and $\operatorname{GKdim} \mathcal{B}(V)=\infty$ by Lemma 2.1 .

As a consequence of Proposition 4.16 we have:

Corollary 4.17. Let $p \in \mathbb{k}^{\times}$such that $p^{4} \neq 1$. Assume that $q_{11}=p$ and $\widetilde{q}_{12}=q_{22}=p^{4}$. Then $\operatorname{GKdim} \mathcal{B}(V)=\infty$.

Proof. If $p$ is a root of 1 and $p^{4} \neq 1$, then $V$ is of Cartan type. Let $A=$ $\left(a_{i j}\right)_{i, j \in \mathbb{I}_{2}}$ be the Cartan matrix of $V$. Recall that $m \alpha_{1}+\alpha_{2}$ is a root if and only if $0 \leq m \leq-a_{12}$. We study three cases according with the order of $p$ :

- If $p \in \mathbb{G}_{4 N}^{\prime}, N \geq 2$, then $-a_{12}=4 N-4$. Thus $\gamma=\beta_{2 N-2}$ is a root. As

$$
q_{\gamma \gamma}=q_{11}^{(2 N-2)^{2}} \widetilde{q}_{12}^{2 N-2} q_{22}=p^{4 N^{2}}=1,
$$

$\operatorname{GKdim} \mathcal{B}(V)=\infty$ by Proposition 4.16 
- If $p \in \mathbb{G}_{4 N+2}^{\prime}, N \geq 1$, then $-a_{12}=4 N-2$. In particular, $\gamma=\beta_{2 N-1}$ is a root. Now $2 \beta_{2 N-1}$ is a root by Lemma 4.11 since $y_{2 N} \neq 0$ and

$$
p_{2 N-1}=q_{\gamma \gamma}=q_{11}^{(2 N-1)^{2}} \widetilde{q}_{12}^{2 N-1} q_{22}=p^{(2 N+1)^{2}}=-1 .
$$

Thus $\operatorname{GKdim} \mathcal{B}(V)=\infty$ by Proposition 4.16 .

- If $p \in \mathbb{G}_{2 N+1}^{\prime}, N \geq 1$, then $-a_{21}=2 N$. Thus $\gamma=N \alpha_{2}+\alpha_{1}$ is a root, and

$$
q_{\gamma \gamma}=q_{22}^{N^{2}} \widetilde{q}_{12}^{N} q_{11}=p^{(2 N+1)^{2}}=1,
$$

$\operatorname{GKdim} \mathcal{B}(V)=\infty$, again by Proposition 4.16.

Finally, if $p$ is not a root of 1 , then $\mathcal{B}(V)$ does not admit all reflections and hence $\operatorname{GKdim} \mathcal{B}(V)=\infty$.

We apply next Corollary 4.17in the braided Hopf algebra $K_{\geq m+1} / K_{>m+1}$. Since $x_{1}^{k} \in K_{>m}$ for all $k, m \in \mathbb{N}$, (2.4) implies that $y_{m} \in K_{\geq m}$ is a primitive element of $\mathbb{N}_{0}^{2}$-degree $\beta_{m}$ in $K_{\geq m} / K_{>m}$ for all $m \in \mathbb{N}$. Then (2.4) and (2.4) leads to the following shape of the coproduct of $w_{m}$ and $y_{m}^{2}$ :

$$
\begin{aligned}
\Delta\left(w_{m}\right) & =w_{m} \otimes 1+1 \otimes w_{m} \\
& +(m+2)_{q_{11}}\left(1-q_{11}^{m+1} \widetilde{q}_{12}\right) q_{\beta_{m+1} \beta_{m}} y_{m+1} \otimes y_{m+1} \\
& + \text { terms } x \otimes y, \operatorname{deg} x=k \alpha_{1}+l \alpha_{2}, k \geq l(m+1)+1 ; \\
\Delta\left(y_{m}^{2}\right) & \in y_{m}^{2} \otimes 1+\left(1+p_{m}\right) y_{m} \otimes y_{m}+1 \otimes y_{m}^{2}+B_{>m} \otimes \mathcal{B}(V) .
\end{aligned}
$$

Assume that $p_{m+1} \neq-1$. By (4.11) and (4.12), $\widetilde{w}_{m}$ is a primitive element of $\mathbb{N}_{0}^{2}$-degree $2 \beta_{m+1}$ in $K_{\geq m+1} / K_{>m+1}$.

Lemma 4.18. Let $m \in \mathbb{N}_{0}$ be such that $p_{m+1} \neq-1$. If $\widetilde{w}_{m} \neq 0$, then $\operatorname{GKdim} \mathcal{B}(V)=\infty$.

Proof. The subalgebra of $K_{\geq m+1} / K_{>m+1}$ generated by $y_{m+1}$ and $\widetilde{w}_{m}$ is a pre-Nichols algebra of diagonal type. Let $W$ be the $\mathbb{k}$-span of $y_{m+1}$ and $\widetilde{w}_{m}$, $p=q_{\beta_{m+1} \beta_{m+1}}$. The braiding matrix of $W$ is

$$
\left(\begin{array}{cc}
p & p^{2} \\
p^{2} & p^{4}
\end{array}\right)
$$

If $p^{4}=1$ then $2 \beta_{m+1}$ is a root of $V$ of infinite height. Thus $\operatorname{GKdim} \mathcal{B}(V)=$ $\infty$ by Proposition 4.16.

If $p^{4} \neq 1$, then $W$ satisfies the assumptions of Corollary 4.17 and hence $\operatorname{GKdim} \mathcal{B}(W)=\infty$. Since $\mathcal{B}(W)$ is a subquotient of $K_{\geq m+1} / K_{>m+1}$, and this is a subquotient of $\mathcal{B}(V)$, we have that $\operatorname{GKdim} \mathcal{B}(V)=\infty$.

We now apply Lemma 4.18 combined with the Lemmas in 4.1

Lemma 4.19. Assume that

$$
\widetilde{q}_{12} \neq 1, \quad(2)_{q_{11}}\left(1-q_{11} \widetilde{q}_{12}\right) \neq 0, \quad(2)_{q_{22}}\left(1-\widetilde{q}_{12} q_{22}\right) \neq 0, \quad q_{11} \widetilde{q}_{12}^{2} q_{22} \neq-1 .
$$

Then $\operatorname{GKdim} \mathcal{B}(V)=\infty$. 
Proof. By Lemma 2.2, $y_{2} \neq 0$. If $p_{1}=-1$, then $2 \beta_{1}$ is a root of $V$ by Lemma 4.11, so $\operatorname{GKdim} \mathcal{B}(V)=\infty$ by Proposition 4.16 .

Assume now that $p_{1} \neq-1$. Then

$$
\Delta\left(y_{1}^{2}\right)=y_{1}^{2} \otimes 1+1 \otimes y_{1}^{2}+\left(1+p_{1}\right) y_{1} \otimes y_{1}
$$

in $K_{\geq 1} / K_{>1}$ and hence $\widetilde{w}_{0}$ is primitive in $K_{\geq 1} / K_{>1}$. By Lemma 4.14 $\widetilde{w}_{0}$ is non-zero. Now we apply Lemma 4.18 .

Without loss of generality, we assume that $\left|a_{12}^{V}\right| \geq\left|a_{21}^{V}\right|>0$. We find a bound for $a_{12}^{V}, a_{21}^{V}$ to reduce the possibilities.

Lemma 4.20. Let $V$ be a braided vector space of diagonal type and dimension 2 such that $a_{12}, a_{21} \leq-3$. Then $\operatorname{GKdim} \mathcal{B}(V)=\infty$.

Proof. Suppose that $\operatorname{GKdim} \mathcal{B}(V)<\infty$. Then $\widetilde{w}_{0}=0$, so by Lemma 4.14 we have that $q_{11} \widetilde{q}_{12}^{2} q_{22}=-1$. As $y_{3} \neq 0$, Lemma 4.15) implies that $\left(1-q_{11}^{3} \widetilde{q}_{12}\right)\left(1+q_{11}^{2}\right)=0$, so $a_{12}=-3$ and analogously $a_{21}=-3$. We exclude the case $q_{11}^{2}=q_{22}^{2}=-1$ : if this happens, then $1=q_{11}^{2} \widetilde{q}_{12}^{4} q_{22}^{2}=\widetilde{q}_{12}^{4}$, so $\widetilde{q}_{12} \in \mathbb{G}_{4}$, but this gives a contradiction since $a_{12}^{V}=a_{21}^{V}=-3$. Hence we may assume $q_{22}^{3} \widetilde{q}_{12}=1$. Let $r=q_{22}$, so $\widetilde{q}_{12}=r^{-3}$. As $-1=q_{11} \widetilde{q}_{12}^{2} q_{22}=r^{-5} q_{11}$, we have that $q_{11}=-r^{5}$.

First we assume that $q_{11}^{3} \widetilde{q}_{12}=1$. Hence $q_{11}^{3}=r^{3}$ so either $q_{11}=r \in \mathbb{G}_{8}^{\prime}$ or $q_{11}=r^{17} \in \mathbb{G}_{24}^{\prime}$. We compute $d_{t}$ as in (4.6) when $n=1$, for each case:

- If $q_{11}=r \in \mathbb{G}_{8}^{\prime}$, then $p_{1}=r^{7}$, so $N=8$ and

$$
\begin{aligned}
d_{t} & =1-r^{7 t+6}+\frac{r^{7 t}\left(1-r^{-2}\right)(1+r)}{(t)_{r^{7}}} \\
& =1-r^{7 t+6}+\frac{r^{7 t-3}\left(r^{2}-1\right)^{2}}{1-r^{7 t}}=1-r^{7 t+6}+\frac{2 r^{7 t+3}}{1-r^{7 t}} .
\end{aligned}
$$

Hence $d_{t}=0 \Longleftrightarrow\left(1-r^{7 t+6}\right)\left(1-r^{7 t}\right)=2 r^{7 t+7}$. Now we check the validity of this equation for $1 \leq t \leq N-2=6$ :

$$
\begin{aligned}
t & =1: & \left(1-r^{5}\right)\left(1-r^{7}\right) & \neq 2 r^{6} ; \\
t & =2: & \left(1-r^{4}\right)\left(1-r^{6}\right)=2\left(1-r^{6}\right) & \neq 2 r^{5} ; \\
t & =3: & \left(1-r^{3}\right)\left(1-r^{5}\right) & \neq 2 r^{4} ; \\
t & =4: & \left(1-r^{2}\right)\left(1-r^{4}\right)=2\left(1-r^{2}\right) & \neq 2 r^{3} ; \\
t & =5: & (1-r)\left(1-r^{3}\right) & \neq 2 r^{2} ; \\
t & =6: & (1-1)\left(1-r^{2}\right)=0 & \neq 2 r .
\end{aligned}
$$

Thus $d_{t} \neq 0$ for all $1 \leq t \leq 6$ so $y_{1}^{8} \neq 0$ by Proposition 4.8 , and hence $8 \beta_{1}$ is a root by Lemma 4.9. This implies that $\operatorname{GKdim} \mathcal{B}(V)=\infty$ by Proposition 4.16 .

- If $q_{11}=r^{17} \in \mathbb{G}_{24}^{\prime}$, then $p_{1}=r^{15}$, so $N=8$ and

$$
d_{t}=1-r^{15 t-2}+\frac{r^{15 t}\left(1+r^{2}\right)\left(1+r^{17}\right)\left(1+r^{3}\right)}{1-r^{15 t}} .
$$


Hence $d_{t}=0 \Longleftrightarrow\left(1-r^{15 t-2}\right)\left(1-r^{15 t}\right)=-r^{15 t}\left(1+r^{2}\right)\left(1+r^{17}\right)\left(1+r^{3}\right)$.

Now we check the validity of this equation for $1 \leq t \leq N-2=6$ :

$$
\begin{aligned}
& t=1: \quad\left(1-r^{13}\right)\left(1-r^{15}\right) \neq r^{3}\left(1+r^{2}\right)\left(1+r^{17}\right)\left(1+r^{3}\right) ; \\
& t=2: \quad\left(1-r^{4}\right)\left(1-r^{6}\right) \neq r^{18}\left(1+r^{2}\right)\left(1+r^{17}\right)\left(1+r^{3}\right) \text {; } \\
& t=3: \quad\left(1-r^{19}\right)\left(1-r^{21}\right) \neq r^{9}\left(1+r^{2}\right)\left(1+r^{17}\right)\left(1+r^{3}\right) ; \\
& t=4: \quad 2\left(1-r^{10}\right) \neq\left(1+r^{2}\right)\left(1+r^{17}\right)\left(1+r^{3}\right) ; \\
& t=5: \quad(1-r)\left(1-r^{3}\right) \neq r^{15}\left(1+r^{2}\right)\left(1+r^{17}\right)\left(1+r^{3}\right) ; \\
& t=6: \quad\left(1-r^{16}\right)\left(1-r^{18}\right) \neq r^{6}\left(1+r^{2}\right)\left(1+r^{17}\right)\left(1+r^{3}\right) .
\end{aligned}
$$

Thus $d_{t} \neq 0$ for all $1 \leq t \leq 6$ so $y_{1}^{8} \neq 0$ by Proposition 4.8 , and hence $8 \beta_{1}$ is a root by Lemma 4.9. This implies that $\operatorname{GKdim} \mathcal{B}(V)=\infty$ by Proposition 4.16 .

The last case is $q_{11} \in \mathbb{G}_{4}^{\prime}$. Thus $r^{5}=-q_{11}$. As $a_{12}^{V}=-3$ we have that $r \in \mathbb{G}_{20}^{\prime}$. Again we compute $d_{t}$ for $n=1$. Here, $p_{1}=r^{13}$, so $N=20$ and

$$
d_{t}=1-r^{13 t}+\frac{r^{13 t}\left(1+r^{2}\right)\left(1+r^{15}\right)\left(1+r^{3}\right)}{1-r^{13 t}} .
$$

Hence $d_{t}=0 \Longleftrightarrow\left(1-r^{13 t}\right)^{2}=r^{13 t+5}\left(1+r^{2}\right)\left(1+r^{5}\right)\left(1+r^{3}\right)$. Now we check the validity of this equation for $1 \leq t \leq N-2=18$ :

$$
\begin{array}{lrl}
t=1: & & \left(1+r^{3}\right)^{2} \neq r^{18}\left(1+r^{2}\right)\left(1+r^{5}\right)\left(1+r^{3}\right) ; \\
t=2: & & \left(1-r^{6}\right)^{2} \neq r^{11}\left(1+r^{2}\right)\left(1+r^{5}\right)\left(1+r^{3}\right) ; \\
t=3: & & \left(1+r^{9}\right)^{2} \neq r^{4}\left(1+r^{2}\right)\left(1+r^{5}\right)\left(1+r^{3}\right) ; \\
t=4: & & \left(1+r^{2}\right)^{2} \neq r^{17}\left(1+r^{2}\right)\left(1+r^{5}\right)\left(1+r^{3}\right) ; \\
t=5: & & 2 r^{15} \neq r^{10}\left(1+r^{2}\right)\left(1+r^{5}\right)\left(1+r^{3}\right) ; \\
t=6: & \left(1+r^{8}\right)^{2} \neq r^{3}\left(1+r^{2}\right)\left(1+r^{5}\right)\left(1+r^{3}\right) ; \\
t=7: & \left(1+r^{2} \neq r^{16}\left(1+r^{2}\right)\left(1+r^{5}\right)\left(1+r^{3}\right) ;\right. \\
t=8: & \left(1+r^{7}\right)^{2} \neq r^{2}\left(1+r^{2}\right)\left(1+r^{5}\right)\left(1+r^{3}\right) ; \\
t=9: & \left(1-r^{3}\right)^{2} \neq r^{8}\left(1+r^{2}\right)\left(1+r^{5}\right)\left(1+r^{3}\right) ; \\
t=10: & \left(1-r^{9}\right)^{2} \neq r^{14}\left(1+r^{2}\right)\left(1+r^{5}\right)\left(1+r^{3}\right) ; \\
t=11: & \left(1-r^{2}\right)^{2} \neq r^{7}\left(1+r^{2}\right)\left(1+r^{5}\right)\left(1+r^{3}\right) ; \\
t=12: & 2 r^{5} \neq\left(1+r^{2}\right)\left(1+r^{5}\right)\left(1+r^{3}\right) ; \\
t=14: & \left(1-r^{8}\right)^{2} \neq r^{13}\left(1+r^{2}\right)\left(1+r^{5}\right)\left(1+r^{3}\right) ; \\
t=15: & (1-r)^{2} \neq r^{6}\left(1+r^{2}\right)\left(1+r^{5}\right)\left(1+r^{3}\right) ; \\
t=16: &
\end{array}
$$




$$
t=18: \quad\left(1+r^{4}\right)^{2} \neq r^{19}\left(1+r^{2}\right)\left(1+r^{5}\right)\left(1+r^{3}\right) .
$$

Thus $d_{t} \neq 0$ for all $1 \leq t \leq 18$ so $y_{1}^{20} \neq 0$ by Proposition 4.8 , and hence $20 \beta_{1}$ is a root by Lemma 4.9, Again, $\operatorname{GK} \operatorname{dim} \mathcal{B}(V)=\infty$ by Proposition 4.16,

We finally assume that $\mathcal{B}(V)$ has finite GK-dimension. By Remark 2.5, $V$ admits all reflections. We consider all possible cases with $a_{21} \in\{-1,-2\}$, not covered by previous arguments, and conclude that the root system is finite-i. e. the Dynkin diagram appears in [H2, Table 1].

4.2.1. $a_{12}^{V}=a_{21}^{V}=-1$. We have that

$$
\left(q_{11} \widetilde{q}_{12}-1\right)(2)_{q_{11}}=0, \quad\left(q_{22} \widetilde{q}_{12}-1\right)(2)_{q_{22}}=0 .
$$

The four possible diagrams appear in [H2, Table 1, Rows 1 \& 2].

4.2.2. $a_{12}^{V}=-2, a_{21}^{V}=-1$. We have that

$$
\left(q_{11}^{2} \widetilde{q}_{12}-1\right)(3)_{q_{11}}=0, \quad\left(q_{22} \widetilde{q}_{12}-1\right)(2)_{q_{22}}=0 .
$$

If $q_{22} \widetilde{q}_{12}=1$, then we have [H2, Table 1 , Rows $3 \& 5$ ].

Now we assume that $q_{22}=-1$. If $q_{11}^{2} \widetilde{q}_{12}=1$, then we get [H2, Table 1 , Row 4]. Let $q_{11} \in \mathbb{G}_{3}^{\prime}$. For simplicity we set $q=q_{11}, r=\widetilde{q}_{12}$. Let $\left(t_{i j}\right)_{i, j \in \mathbb{I}}$ be the braiding matrix of $\mathcal{R}^{2}(V)$ : its Dynkin diagram is $\stackrel{-q r}{0} \stackrel{r^{-1}}{-1} \stackrel{-1}{\circ}$. We study the possible values of $a:=a_{12}^{\mathcal{R}^{2}(V)}$. Notice that $a \leq-2$.

$\circ a=-2$. If $1=t_{11}^{2} \widetilde{t}_{12}=q^{2} r$, then $V$ appears in [H2, Table 1, Row 4]. Otherwise $1=t_{11}^{3}=-r^{3}$, so $r=-q^{ \pm 1}$. As $1 \neq t_{11}=-q r$, we have that $r=-q$ and $V$ appears in [H2, Table 1, Row 6].

$\circ a=-3$. Either $1=t_{11}^{3} \widetilde{t}_{12}=-r^{2}$, in which case $r \in \mathbb{G}_{4}^{\prime}$ and $V$ appears in [H2, Table 1, Row 8], or else $1=t_{11}^{4}=q r^{4}$, in which case $r \in \mathbb{G}_{12}^{\prime}$ and $V$ appears in [H2, Table 1, Row 7].

$\circ a \leq-4$. Notice that $\widetilde{w}_{0}=0$ by Lemma 4.14, and $\widetilde{w}_{1}=0$ by Lemma 4.15 (a) since $-t_{11} \widetilde{t}_{12}=q \in \mathbb{G}_{3}^{\prime}$. Hence we may apply Lemma 4.12, as $\widetilde{w}_{2}=0$, the scalar in Remark 4.13 (v) is zero. That is,

$$
0=\left(1+q^{2} r^{5}\right)\left(1-q r^{3}\right)\left(1+q^{2} r^{2}\right)\left(1+q^{2} r^{4}\right) .
$$

If $q^{2} r^{5}=-1$, then $-r \in \mathbb{G}_{15}^{\prime}$ and $V$ belongs to [H2, Table 1, Row 15]. If $q r^{3}=1$, then $r \in \mathbb{G}_{9}^{\prime}$ and $V$ is in [H2, Table 1, Row 9]. If $q^{2} r^{2}=-1$, then $r \in \mathbb{G}_{12}^{\prime}, q=-r^{2}$ and $V$ belongs to [H2, Table 1, Row 7]. Otherwise $q^{2} r^{4}=-1$, in which case $r \in \mathbb{G}_{24}^{\prime}$ with $q=-r^{4}$, and $V$ is in [H2, Table 1, Row 12].

4.2.3. $a_{12}^{V}=-3, a_{21}^{V}=-1$ with $q_{22}=-1$. First we assume $q_{11}^{3} \widetilde{q}_{12}=1$. Set $q=q_{11}$, so $\widetilde{q}_{12}=q^{-3}$. Let $\left(t_{i j}\right)_{i, j \in \mathbb{I}}$ be the braiding matrix of $\mathcal{R}^{2}(V)$ : its Dynkin diagram is $\stackrel{-q^{-2}}{\circ} \stackrel{q^{3}}{-1} \stackrel{-1}{\circ}$.

○ If $a_{12}^{\mathcal{R}^{2}(V)}=-2, \mathcal{R}^{2}(V)$ appears in 4.2 .2 , so $\mathcal{R}^{2}(V)$ has finite root system and then $V$ too. 
○ If $a_{12}^{\mathcal{R}^{2}(V)}=-3$, then then either $t_{11} \in \mathbb{G}_{4}^{\prime}$, in which case $q \in \mathbb{G}_{8}^{\prime}$ and $V$ belongs to [H2, Table 1 , Row 11]; or else $t_{11}^{3} \widetilde{t}_{12}=1$, in which case $q \in \mathbb{G}_{6}^{\prime}$ and $V$ is of Cartan type $G_{2}$ [H2, Table 1, Row 10].

○ If $a_{12}^{\mathcal{R}^{2}(V)} \leq-4$, then Remark4.13 (v) says that $\left(1+q^{7}\right)\left(1-q^{5}\right)(5)_{-q^{-2}}=0$. If $q^{7}=-1$, then $V$ belongs to [H2, Table 1, Row 16]. If $q^{5}=1$, then $V$ belongs to [H2, Table 1, Row 13]. If $-q^{2} \in \mathbb{G}_{5}^{\prime}$, then $V$ belongs to [H2, Table 1, Row 14].

Finally, if $q_{11}^{3} \widetilde{q}_{12} \neq 1$, then $q_{11}=\eta \in \mathbb{G}_{4}^{\prime}$. Set $q=\widetilde{q}_{12}$. By Lemma 4.15)(a), ○ either $\eta^{3} q^{2}=-1$, so $q^{2}=-\eta$ and $V$ belongs to [H2, Table 1, Row 11]; o or else $-\eta q \in \mathbb{G}_{3}^{\prime}$, in which case $a_{12}^{\mathcal{R}^{2}(V)}=-2, a_{21}^{\mathcal{R}^{2}(V)}=-1$ since the diagram of $\mathcal{R}^{2}(V)$ is $\stackrel{-\eta q}{\circ} \underline{q^{-1}} \stackrel{-1}{\circ}$. Hence $\mathcal{R}^{2}(V)$ appears in 4.2 .2 and thus $\mathcal{R}^{2}(V)$ has finite root system.

4.2.4. $a_{12}^{V}=-3, a_{21}^{V}=-1$ with $q_{22} \widetilde{q}_{12}=1$. If $q_{11}^{3} \widetilde{q}_{12}=1$, then $V$ is of Cartan type $G_{2}$ and the root system is finite [H2, Table 1, Row 10]. Otherwise, $q_{11}=\eta \in \mathbb{G}_{4}^{\prime}$. For simplicity we call $q=q_{22}$ so $\widetilde{q}_{12}=q^{-1}$. Let $\left(t_{i j}\right)_{i, j \in \mathbb{I}}$ be the braiding matrix of $\mathcal{R}^{1}(V)$ : its Dynkin diagram is $\stackrel{\eta}{\circ}-q-q^{-2} \eta$. By Lemma 4.20, $a_{21}^{\mathcal{R}^{1}(V)} \geq-2$.

○ If $a_{21}^{\mathcal{R}^{1}(V)}=-1$, then either $t_{22}=-1$, in which case $q^{2}=-\eta$ and $V$ belongs to [H2, Table 1, Row 11]; or else $t_{22} \widetilde{t}_{12}=1$, in which case $q=-\eta$ and $V$ is of Cartan type $G_{2}$.

○ If $a_{21}^{\mathcal{R}^{1}(V)}=-2$, then either $1=t_{22}^{2} \widetilde{t}_{12}$, in which case $q^{3}=1$, or else $1=t_{22}^{3}$, which implies $q^{6}=-\eta$. For the first case, we compute $p_{1}=q^{-1}$, which has order $N=3$, and by (4.6),$d_{1}=-\eta q \neq 0$. Hence $y_{1}^{3} \neq 0$ by Proposition 4.8. By Lemma 4.9, $3 \beta_{1}$ is a root of $\mathcal{R}^{1}(V)$, thus $\operatorname{GKdim} \mathcal{B}\left(\mathcal{R}^{1}(V)\right)=$ $\infty$ by Proposition 4.16, For the second case, $q \in \mathbb{G}_{24}^{\prime}$ and $V$ belongs to [H2, Table 1, Row 12].

4.2.5. $a_{12}^{V} \leq-4, a_{21}^{V}=-1$ with $q_{22}=-1$. For simplicity we set $q=q_{11}$, $r=\widetilde{q}_{12}$. Let $\left(t_{i j}\right)_{i, j \in \mathbb{I}}$ be the braiding matrix of $\mathcal{R}^{2}(V)$ : its Dynkin diagram is $\stackrel{-q r}{\circ} r^{-1} \stackrel{-1}{\circ}$. As $q^{3} r \neq 1$, Lemma 4.15 (a) says that either $q^{3} r^{2}=-1$ or else $-q r \in \mathbb{G}_{3}^{\prime}$.

○ If $q^{3} r^{2}=-1$, then $t_{11}^{3} \widetilde{t}_{12}=-q^{3} r^{2}=1$ so $a_{12}^{\mathcal{R}^{2}(V)}=-3$. Hence $\mathcal{R}^{2}(V)$ has a finite root system by 4.2 .3 , and $V$ too.

○ If $-q r \in \mathbb{G}_{3}^{\prime}$, then $a_{12}^{\mathcal{R}^{2}(V)}=-2$. Hence $\mathcal{R}^{2}(V)$ has a finite root system by 4.2.2, and $V$ too.

4.2.6. $a_{12}^{V} \leq-4, a_{21}^{V}=-1$ with $q_{22} \widetilde{q}_{12}=1$. For simplicity set $q=q_{11}$, $r=q_{22}$, so $\widetilde{q}_{12}=r^{-1}$. By Lemma 4.15 (b), $t:=-q^{2} r^{-1} \in \mathbb{G}_{3}^{\prime}$ since $a_{12}^{V} \leq-4$. As $y_{4} \neq 0$ we may apply Lemma 4.12 and Remark 4.13 (vii).

$$
0=\left(1-q^{4} r^{-1}\right)\left(1+q^{4} r^{-1}\right)\left(1-q^{5} r^{-1}\right)(3)_{-q}(5)_{q} .
$$


ON FINITE GK-DIMENSIONAL NICHOLS ALGEBRAS OF DIAGONAL TYPE

○ If $q^{4} r^{-1}=1$, then $V$ is of affine Cartan type and $\operatorname{GKdim} \mathcal{B}(V)=\infty$ by Proposition 3.1, a contradiction.

○ If $q^{4} r^{-1}=-1$, then $q^{2}=\left(-q^{4} r^{-1}\right)\left(-q^{2} r^{-1}\right)^{-1}=t^{2}$, so $q= \pm t, r=-t$. If $q=t$, then $a_{12}^{V} \geq-2$; otherwise $q=-t$ and $q_{11} \widetilde{q}_{12}=q r^{-1}=1$ so $a_{12}^{V}=-1$. In any case we obtain a contradiction with $a_{12}^{V} \leq-4$.

○ If $q^{5} r^{-1}=1$, then $q^{3}=-t^{-1} \in \mathbb{G}_{6}^{\prime}$. Hence $-q \in \mathbb{G}_{9}^{\prime}$ and $p_{3}=q^{9} r^{-3} r=$ $q^{-1} \in \mathbb{G}_{18}^{\prime}$. Now we compute $d_{l}$ as in (4.6) for $n=3$ :

$d_{l}=1-q^{-l-1} q^{6} r^{-1}+\frac{q^{-l}\left(1-q^{3} r^{-1}\right)(4)_{q}}{(l)_{q^{-1}}}=1-q^{-l}+\frac{q^{-l}\left(1-q^{-2}\right)(4)_{q}}{(l)_{q^{-1}}}$

Thus $d_{l}=0$ if and only if $q^{l+3}\left(1-q^{-l}\right)^{2}=\left(q^{2}-1\right)\left(q^{4}-1\right)$, but this equality does not hold for $1 \leq l \leq 16$. Hence $18 \beta_{3}$ is a root of $V$ by Proposition 4.8 and Lemma 4.9, so $\operatorname{GKdim} \mathcal{B}(V)=\infty$ by Proposition 4.16.

○ If $-q \in \mathbb{G}_{3}^{\prime}$, then either $t=-q$ or else $t=-q^{-1}$. Both are not possible since $a_{12}^{V} \leq-4$.

○ If $q \in \mathbb{G}_{5}^{\prime}$, then $r=-t^{-1} q^{2} \in \mathbb{G}_{30}^{\prime}$. This is the case in [H2, Table 1, Row $15]$.

4.2.7. $a_{12}^{V}=a_{21}^{V}=-2$. We have that $\left(q_{11} \widetilde{q}_{12}-1\right)(2)_{q_{11}}\left(q_{22} \widetilde{q}_{12}-1\right)(2)_{q_{22}} \neq 0$,

$$
\left(q_{11}^{2} \widetilde{q}_{12}-1\right)(3)_{q_{11}}=0, \quad\left(q_{22}^{2} \widetilde{q}_{12}-1\right)(3)_{q_{22}}=0 .
$$

If $q_{11}^{2} \widetilde{q}_{12}=1=q_{22}^{2} \widetilde{q}_{12}$, then $V$ is of affine Cartan type, a contradiction with Proposition [3.1. Hence we may assume that $q_{11} \in \mathbb{G}_{3}^{\prime}$. By Lemma 4.14, $q_{11} \widetilde{q}_{12}^{2} q_{22}=-1$. Let $\left(t_{i j}\right)_{i, j \in \mathbb{I}}$ be the braiding matrix of $\mathcal{R}^{1}(V)$ :

$$
t_{22}=q_{22} \widetilde{q}_{12}^{2} q_{11}^{4}=q_{22} \widetilde{q}_{12}^{2} q_{11}=-1,
$$

so $\mathcal{R}^{1}(V)$ appears in 4.2 .2 since $\operatorname{GKdim} \mathcal{B}\left(\mathcal{R}^{1}(V)\right)<\infty$. Thus $\mathcal{R}^{1}(V)$ has a finite root system, and $V$ too.

4.2.8. $a_{12}^{V} \leq-3, a_{21}^{V}=-2$. As $\widetilde{w}_{0}=0, q_{11} \widetilde{q}_{12}^{2} q_{22}=-1$ by Lemma 4.14. As $\widetilde{w}_{1}=0$, either $q_{11}^{3} \widetilde{q}_{12}=1$ or else $q_{11}^{2}=-1$ by Lemma 4.15 (c). Hence $a_{12}=-3$. We analyze the possible 4 cases.

If $q_{11}^{3} \widetilde{q}_{12}=1=q_{22}^{2} \widetilde{q}_{12}$, then $V$ is of indefinite Cartan type and

$$
-q_{11}^{2} q_{22}=\left(q_{11} \widetilde{q}_{12}^{2} q_{22}\right) q_{11}^{2} q_{22}=q_{11}^{3} \widetilde{q}_{12} q_{22}^{2} \widetilde{q}_{12}=1,
$$

thus $q_{22}=-q_{11}^{-2}$, and $1=q_{22}^{2} \widetilde{q}_{12}=q_{11}^{-7}$, so $q_{11} \in \mathbb{G}_{7}^{\prime}$. Let $r:=q_{22} \in \mathbb{G}_{14}^{\prime}$, so $q_{11}=r^{10}, \widetilde{q}_{12}=r^{12}$. We compute $d_{t}$ as in (4.6) for $n=1$. Here, $p_{1}=r^{9}$, so $N=14$ and

$$
d_{t}=1-r^{9 t+13}+\frac{r^{9 t}(1+r)\left(1-r^{3}\right)\left(1+r^{2}\right)}{1-r^{9 t}} .
$$

Hence $d_{t}=0 \Longleftrightarrow\left(1-r^{9 t+13}\right)\left(1-r^{9 t}\right)=r^{9 t}(1+r)\left(r^{3}-1\right)\left(1+r^{2}\right)$. Now we check the validity of this equation for $1 \leq t \leq N-2=12$ :

$$
\begin{array}{ll}
t=1: & (1+r)\left(1+r^{2}\right) \neq r^{9}(1+r)\left(r^{3}-1\right)\left(1+r^{2}\right) ; \\
t=2: & \left(1-r^{3}\right)\left(1-r^{4}\right) \neq r^{4}(1+r)\left(r^{3}-1\right)\left(1+r^{2}\right) ;
\end{array}
$$




$$
\begin{array}{rlrl}
t=3: & \left(1+r^{5}\right)\left(1+r^{6}\right) \neq r^{13}(1+r)\left(r^{3}-1\right)\left(1+r^{2}\right) ; \\
t=4: & 2(1+r) \neq r^{8}(1+r)\left(r^{3}-1\right)\left(1+r^{2}\right) ; \\
t=5: & \left(1-r^{2}\right)\left(1-r^{3}\right) \neq r^{3}(1+r)\left(r^{3}-1\right)\left(1+r^{2}\right) ; \\
t=6: & \left(1+r^{4}\right)\left(1+r^{6}\right) \neq r^{12}(1+r)\left(r^{3}-1\right)\left(1+r^{2}\right) ; \\
t=7: & 2\left(1-r^{6}\right) \neq-(1+r)\left(r^{3}-1\right)\left(1+r^{2}\right) ; \\
t=8: & (1-r)\left(1-r^{2}\right) \neq r^{2}(1+r)\left(r^{3}-1\right)\left(1+r^{2}\right) ; \\
t=9: & \left(1+r^{3}\right)\left(1+r^{4}\right) \neq r^{11}(1+r)\left(r^{3}-1\right)\left(1+r^{2}\right) ; \\
t=10: & \left(1-r^{5}\right)\left(1-r^{6}\right) \neq r^{6}(1+r)\left(r^{3}-1\right)\left(1+r^{2}\right) ; \\
t=11: & 0 & \neq r(1+r)\left(r^{3}-1\right)\left(1+r^{2}\right) ; \\
t=12: & \left(1+r^{2}\right)\left(1+r^{3}\right) \neq r^{10}(1+r)\left(r^{3}-1\right)\left(1+r^{2}\right) .
\end{array}
$$

Thus $d_{t} \neq 0$ for all $1 \leq t \leq 12$ so $y_{1}^{14} \neq 0$ by Proposition 4.8 , and hence $14 \beta_{1}$ is a root by Lemma 4.9. Therefore $\operatorname{GKdim} \mathcal{B}(V)=\infty$ by Proposition 4.16.

Assume that $q_{11}=\eta \in \mathbb{G}_{4}^{\prime}, q_{22}^{2} \widetilde{q}_{12}=1$ : to simplify the notation, set $q=q_{22}$, so $\widetilde{q}_{12}=q^{-2}$. Then $-1=q_{11} \widetilde{q}_{12}^{2} q_{22}=\eta q^{-3}$, so $q^{3}=-\eta$. The matrix $\left(t_{i j}\right)_{i, j \in \mathbb{I}}$ of $\mathcal{R}^{1}(V)$ has diagram $\stackrel{\eta}{\circ}-q^{2}-q^{-2}$. Thus $a_{21}^{\mathcal{R}^{1}(V)}=-1$ and then $\mathcal{R}^{1}(V)$ has finite root system, since $\operatorname{GKdim} \mathcal{B}\left(\mathcal{R}^{1}(V)\right)=\operatorname{GKdim} \mathcal{B}(V)<\infty$.

Assume now $q_{11}^{3} \widetilde{q}_{12}=1, q_{22}=\zeta \in \mathbb{G}_{3}^{\prime}$. Let $q=q_{11}$ so $\widetilde{q}_{12}=q^{-3}$. The matrix $\left(t_{i j}\right)_{i, j \in \mathbb{I}}$ of $\mathcal{R}^{2}(V)$ has diagram $\stackrel{-1}{\circ} \underline{\zeta^{2} q^{3}} \stackrel{\zeta}{\circ}$. Thus $a_{12}^{\mathcal{R}^{1}(V)}=-1$ and then $\mathcal{R}^{2}(V)$ has finite root system, since $\operatorname{GKdim} \mathcal{B}\left(\mathcal{R}^{2}(V)\right)=\operatorname{GKdim} \mathcal{B}(V)<\infty$.

If $q_{11}=\eta \in \mathbb{G}_{4}^{\prime}, q_{22}=\zeta \in \mathbb{G}_{3}^{\prime}$, then $-1=q_{11} \widetilde{q}_{12}^{2} q_{22}=\eta q^{2} \zeta$, where $q=\widetilde{q}_{12}$.

Hence the matrix $\left(t_{i j}\right)_{i, j \in \mathbb{I}}$ of $\mathcal{R}^{2}(V)$ has diagram $\stackrel{-1}{\circ} \underline{q^{-1} \zeta^{2}} \zeta$. Thus $\mathcal{R}^{2}(V)$ has finite root system, and $V$ too.

\section{REFERENCES}

[A] N. Andruskiewitsch. An Introduction to Nichols Algebras. In Quantization, Geometry and Noncommutative Structures in Mathematics and Physics. A. Cardona, P. Morales, H. Ocampo, S. Paycha, A. Reyes, eds., Springer (2017), 135-195.

[AA1] N. Andruskiewitsch, I. Angiono. On Nichols algebras with generic braiding, in Modules and Comodules, T. Brzezinski; J. L. Gómez Pardo; I. Shestakov; P. F. Smith (Eds.). Trends in Mathematics (2008), 47-64.

[AA2] N. Andruskiewitsch, I. Angiono. On Finite dimensional Nichols algebras of diagonal type. Bull. Math. Sci. 7 (2017), 353-573.

[AAH] N. Andruskiewitsch, I. Angiono, I. Heckenberger. On finite GK-dimensional Nichols algebras over abelian groups. arXiv:1606.02521

[AS1] N. Andruskiewitsch, H.-J. Schneider. Pointed Hopf algebras, New directions in Hopf algebras, MSRI series Cambridge Univ. Press (2002), 1-68 .

[An1] I. Angiono. A presentation by generators and relations of Nichols algebras of diagonal type and convex orders on root systems, J. Europ. Math. Soc. 17 (2015), 2643-2671. 
[C+] L. Carbone, S. Chung, C. Cobbs, R. McRae, D. Nandi, Y. Naqvi, D. Penta. Classification of hyperbolic Dynkin diagrams, root lengths and Weyl group orbits. J. Phys. A: Math. Theor. 43 (2010) (15): 155209.

$[\mathrm{CH}]$ M. Cuntz, I. Heckenberger. Weyl groupoids with at most three objects. J. Pure Appl. Algebra 213 (2009), 1112-1128.

[GH] M. Graña, I. Heckenberger. On a factorization of graded Hopf algebras using Lyndon words. J. Algebra 314 (2007), 324-343.

[H1] I. Heckenberger. The Weyl groupoid of a Nichols algebra of diagonal type, Inventiones Math. 164 (2006), 175-188.

[H2] I. Heckenberger. Classification of arithmetic root systems, Adv. Math. 220 (2009), 59-124.

[HS] I. Heckenberger, H.-J. Schneider. Root systems and Weyl groupoids for Nichols algebras, Proc. London Math. Soc. 101 (2010), 623-654.

[Kh] V. Kharchenko. A quantum analog of the Poincare-Birkhoff-Witt theorem, Algebra and Logic 38 (1999), 259-276.

[K] V. Kac. Infinite-dimensional Lie algebras. Third edition. Cambridge University Press, Cambridge, 1990. xxii+400 pp.

[KL] G. Krause, T. Lenagan,. Growth of algebras and Gelfand-Kirillov dimension. Revised edition. Graduate Studies in Mathematics, 22. American Mathematical Society, Providence, RI, 2000. $\mathrm{x}+212 \mathrm{pp}$

[Mo] S. Montgomery. Hopf algebras and their action on rings, CBMS Regional Conference Series 82 (1993).

[R] M. Rosso. Quantum groups and quantum shuffles. Invent. Math. 133 (1998), 399416.

[Y] H. Yamane. Representations of a $\mathbb{Z} / 3 \mathbb{Z}$-quantum group. Publ. Res. Inst. Math. Sci. 43 (2007), 75-93.

FamaF-CiEm (CONiCET), Universidad Nacional de Córdoba, Medina Allende s/n, Ciudad Universitaria, 5000 Córdoba, República Argentina.

E-mail address: (andrus|angiono)@famaf.unc.edu.ar

Philipps-Universität Marburg, Fachbereich Mathematik und Informatik, HansMeerwein-Strasse, D-35032 Marburg, Germany.

E-mail address: heckenberger@mathematik.uni-marburg.de 